\title{
Establishment in a new habitat by polygenic adaptation
}

\author{
N. H. Barton ${ }^{\mathrm{a}, 1}$, A. M. Etheridge ${ }^{\mathrm{b}}$ \\ ${ }^{a}$ Institute of Science and Technology \\ Am Campus I \\ A-3400 Klosterneuberg \\ Austria \\ ${ }^{b}$ Department of Statistics \\ University of Oxford \\ 24-29 St Giles \\ Oxford OX1 $3 L B$ \\ $U K$
}

\begin{abstract}
Maladapted individuals can only colonise a new habitat if they can evolve a positive growth rate fast enough to avoid extinction, a process known as evolutionary rescue. We treat log fitness at low density in the new habitat as a single polygenic trait and use the infinitesimal model to follow the evolution of the growth rate; this assumes that the trait values of offspring of a sexual union are normally distributed around the mean of the parents' trait values, with variance that depends only on the parents' relatedness. The probability that a single migrant can establish depends on just two parameters: the mean and genetic variance of the trait in the source population. The chance of success becomes small if migrants come from a population with mean growth rate in the new habitat more than a few standard deviations below zero; this chance depends roughly equally on the probability that the initial founder is unusually fit, and on the subsequent increase in growth rate of its offspring as a result of selection. The loss of genetic variation during the founding event is substantial, but highly variable. With continued migration at rate $M$, establishment is inevitable; when migration is rare, the expected time to establishment decreases inversely with $M$. However, above a threshold migration rate, the population may be trapped in a 'sink' state, in which adaptation is held back by gene flow; above this threshold, the expected time to establishment increases exponentially with $M$. This threshold behaviour
\end{abstract}

Email addresses: n.barton@ist.ac.at (N. H. Barton), etheridg@stats.ox.ac.uk (A. M. Etheridge)

${ }^{1}$ Work supported by European Research Council grant 250152 
is captured by a deterministic approximation, which assumes a Gaussian distribution of the trait in the founder population with mean and variance evolving deterministically. By assuming a constant genetic variance, we also develop a diffusion approximation for the joint distribution of population size and trait mean, which extends to include stabilising selection and density regulation. Divergence of the population from its ancestors causes partial reproductive isolation, which we measure through the reproductive value of migrants into the newly established population.

Keywords: Local adaptation, infinitesimal model, evolutionary rescue, parapatric speciation, quantitative genetics, diffusion, migration load

\section{Introduction}

Can a population establish itself in a new habitat, adapting to the new conditions despite random drift and gene flow? This question is relevant in several contexts: the evolution of specialist host races, perhaps eventually leading to speciation; at the edge of a species' range, where adaptation to extreme conditions is necessary; and 'evolutionary rescue', following a catastrophic change in environment, or loss of adaptation by random drift (Gomulkiewicz and Holt, 1995; Kawecki, 2008).

In this paper, we focus on the initial establishment of a population by migrants, and its subsequent reproductive isolation. The source population is poorly adapted to the new environment, and would, on average, decline to extinction in the new conditions. However, migrants and their offspring may by chance be better adapted, and moreover, selection will cause further adaptation. Provided that gene flow is not so high as to swamp the incipient adaptation, growth rates may become positive, and a large, well-adapted population may be established.

As the population grows, the proportion of migrants decreases, as does random drift; these two positive feedbacks ensure rapid and secure establishment, that will ultimately be limited by density-dependent regulation of population size, and by stabilising selection on the trait, towards a new optimum. During establishment, we can ignore these two processes, focussing on the initial adaptation and growth via directional selection from low density (Holt and Gomulkiewicz, 1997). Subsequent evolution, perhaps based on new mutations, may allow further adaptation, and will lead to speciation if divergence (both adaptive and non-adaptive) is sufficient to cause strong reproductive isolation. 
This problem has received considerable attention in recent years, since it is relevant to survival of endangered populations, delimitation of the species' range, and invasion of non-native species (Gomulkiewicz et al., 2010; Gomulkiewicz and Shaw, 2013). The positive feedback between adaptation and population size was identified as a factor that can limit species' range by Haldane (1956); the consequent trapping of populations in a maladapted 'sink' state has been studied both in single demes (e.g. Tufto, 2001; Holt et al., 2003; Holt and Barfield, 2011) and along one-dimensional environmental gradients (Kirkpatrick and Barton, 1997; Polechová and Barton, 2015). Such analyses have almost all been based on simulations of discrete loci, or on deterministic models that assume a constant additive variance for one or more traits. Tufto $(2000,2001)$ applied the infinitesimal model to study establishment despite initial maladaptation. His approach allows the evolution of trait variance due to selection and migration. We develop this model, taking account of random drift and demographic stochasticity.

The infinitesimal model motivates a deterministic approximation for the evolution of population size and trait mean, similar to that considered by Tufto (2001). (In an appendix, we show that inbreeding can also be incorporated into the approximation in a relatively straightforward way.) We extend this deterministic approximation to a diffusion approximation that takes account of random sampling drift in the trait mean and demographic stochasticity.

The central result of our analyses of these models takes a remarkably simple form: establishment depends upon just two dimensionless parameters, the change in trait needed to make growth positive and the fitness of an average migrant, both being expressed relative to the additive variance in the source population.

Once the new population is established, we can use the same approximation to calculate its reproductive isolation from the source, measured through the reproductive value of an individual backcrossing into the population. We show that once again this depends on very few parameters: the divergence of the new population from its source and its degree of inbreeding.

Adaptation to a new environment may sometimes involve one or a few changes of major effect - for example, a change in preference for a new host, or origin of a distinct and reproductively isolated polyploid. However, we deal here with the more common, and more challenging, case, where adaptation is polygenic, possibly acting via many biological mechanisms. We can simplify drastically by thinking of a single polygenic trait - log fitness at low density in the new habitat - which we take to be under directional selection. As argued by Holt and Gomulkiewicz (1997), at low density it is the loga- 
rithm of the absolute fitness that will determine whether a population will increase. This fitness may be mediated via very many traits, but we absorb the genetic component of all of these into a single value, determined by the additive effect of many genes. This is less restrictive than it might seem. Even with strong underlying interactions at the level of genes, trait variance may be largely additive (Hill et al. 2008). There is little direct evidence on fitness variance, but indirect arguments suggest that there is substantial additive variance (Charlesworth, 2015).

Our approach may seem paradoxical, since existing theory apparently shows that gene flow should swamp polygenic adaptation. By analogy with the mutation load, migration reduces fitness by an amount equal to the mutation rate, for each locally adapted locus. If alleles at different loci are in linkage equilibrium (as will be the case if migration is much weaker than total selection), then the migration load is proportional to the number of locally adapted loci, and may become disastrously high (Kirkpatrick and Barton, 2006). Looking at the problem in another way, selection at each locus must be stronger than migration to prevent local alleles from being swamped by gene flow, which implies that alleles of small effect cannot contribute to local adaptation.

However, these arguments only apply if locally favoured alleles are absent or very rare in the source population. If adaptation is instead based on standing variation, then alleles of indefinitely small effect can contribute (Le Corre and Kremer, 2012; Yeaman, 2015). To see this, consider an additive trait in a population of diploids, with two possible alleles at locus $i$, having effect $\pm \alpha_{i}$ on the trait value. Writing $p_{i}$ for the proportion of alleles of the first type in the new environment and $p_{i}^{\prime}$ for the corresponding proportion in the source population, migration contributes $m\left(p_{i}^{\prime}-p_{i}\right)$ to the rate of change of $p_{i}$, but a selection gradient $\beta$ on the trait causes selection $\beta \alpha_{i}$, and so contributes $\beta \alpha p_{i}\left(1-p_{i}\right)$. Thus selection can maintain a difference in allele frequency $\beta \alpha_{i} p_{i} q_{i} / m$ in migration selection balance. Summing over loci, we find a difference in trait mean between the new environment and the source population of

$$
\Delta \bar{z}=\frac{\beta}{m} \sum_{i} 2 \alpha_{i}^{2} p_{i} q_{i}=\frac{\beta}{m} V_{A},
$$

where $V_{A}$ is the additive genetic variance. This result can of course be obtained by a purely phenotypic argument, and is independent of the number and effects of individual loci. The difference in allele frequency at each locus may be small, but the cumulative effect can be large, shifting a set of traits by many standard deviations, to well outside their original range. 
A similar argument holds when random drift is included: slight changes in the neutral distribution of allele frequencies can have large effects on phenotype, even if drift and migration dominate selection at individual loci (Robertson, 1960; Kimura, 1981). We will consider the limiting infinitesimal model, which assumes a very large number of unlinked loci with additive effects. Then, the evolution of the trait distribution depends essentially only on the variance between offspring that is released by recombination. Within each family, the trait values of the offspring are normally distributed, with mean equal to the mid-parent value, and fixed variance $V$ if the parents are unrelated. In a finite population, the within-family variance decreases in proportion to the parents' relatedness. This simple model has proven remarkably accurate in practical animal breeding (Lynch and Walsh, 1998), in describing artificial selection experiments (Weber and Diggins, 1990), and in analysis of natural populations (Kruuk, 2004). We define the model more carefully below. For a more detailed introduction to the infinitesimal model, its derivation, and its application, we refer to Barton et al. (2017).

Under the infinitesimal model, knowing the trait of an individual provides very little information about the distribution of allele frequencies at any particular locus, which therefore changes only slightly as a result of selection. The sum of effects of slight changes in allele frequency across all the loci can produce a substantial change in the mean, but the within-family variance is determined by the degree of inbreeding of the parents, with the effects of changes in allele frequency only becoming important over a timescale longer by a factor proportional to the number of loci. Under strong selection, the distribution of trait values in the population may rapidly change its variance, and may certainly deviate from normality. However, these changes are due to the cumulative effects of linkage disequilibria amongst the loci, not to changes in the marginal allele frequencies. The establishment and adaptation of a small population under strong selection must occur quickly if it is to avoid extinction, and so we expect the infinitesimal model to be a good approximation to the evolution of polygenic traits.

The rest of this paper is laid out as follows. After defining the model, and its implementation in individual-based simulations, we consider the fate of single migrants, finding (numerically) the probability that they can successfully establish a growing population. We then consider continued migration, and show that there is a threshold fitness of average migrants, above which establishment is rapid. Below this threshold, establishment requires a rare fluctuation in which the migrant is unusually fit. In this regime we also see the swamping effects of gene flow; time to establishment is no longer a monotone function of the migration rate. Assuming that migration rates 
are moderately high and the selection gradient is not too extreme, we then capture the same threshold behaviour through mathematical analysis of a caricature of our model, in which the trait distribution in the new population is assumed Gaussian with mean and variance evolving deterministically. In this regime of continued migration, we also develop a diffusion approximation for the joint evolution of the trait mean with population size, which extends to include stabilising selection and density regulation, and we use it to investigate the stationary distribution of the new population. Close to a stable 'sink' equilibrium, this stationary distribution is the product of three terms (Eq. 10), reflecting the competing effects of migration, directional selection and gene flow. Finally, we derive the reproductive isolation of a well-adapted newly established population, assuming that it is at equilibrium under a balance between mutation and selection and that migration rates from the source are low enough (or the new population is large enough) that the impact of migrants on the equilibrium can be ignored.

The Mathematica notebooks used to generate the examples are provided as supplementary information.

\section{The infinitesimal model}

Individuals are diploid, and may self-fertilise: thus, a single individual can found a new population. Generations are discrete, and do not overlap. We follow the 'breeding value' of a single additive quantitative trait, with individual value $z$. Non-inherited variation is not treated explicitly, but can be thought of as being absorbed into the relation between fitness and breeding value. Migrants (assumed unrelated) come from a large source population, which is in Hardy-Weinberg proportions and linkage equilibrium. The trait is assumed to follow a Gaussian distribution in the source population with mean $\bar{z}_{s}$ and variance $2 \mathrm{~V}$, composed equally of within and between family variance. This corresponds to the equilibrium distribution for a purely additive neutral trait in a large population under the infinitesimal model.

Individuals migrate from the source population, independent of $z$, their number in each generation being Poisson distributed with expectation $M$. Immediately after migration, there are $N(t)$ individuals, which mate randomly, irrespective of whether they are newly arrived or native, and with the possibility of selfing. Offspring are produced, and survive into the next generation with probability that depends on their trait value, and which, if we incorporate population density regulation, will decrease with $N(t)$. The surviving offspring are joined by another Poisson distributed group of immigrants from the source population, resulting in $N(t+1)$ individuals and 
the process repeats.

Although migrants are assumed to be unrelated to each other, and to the native population, the evolving population will become inbred. The variance amongst offspring is the sum of the variance contributed by haploid gametes from each parent, which in turn is generated by segregation at meiosis, in proportion to $\left(1-F_{i i}\right),\left(1-F_{j j}\right)$ where $F_{i i}$ is the probability of identity by descent between the two distinct genes of parent $i$, and similarly for $F_{j j}$. That is, the variance between offspring from diploid parents $i, j$ is

$$
v_{i j}=V\left(1-\frac{\left(F_{i i}+F_{j j}\right)}{2}\right) .
$$

The same formula applies with self-fertilisation.

We model directional selection on the trait by taking fitness to be $w=$ $e^{\beta z}$. The number $N^{*}(t+1)$ in the next generation, before migration, is Poisson distributed, with expectation $N(t) \bar{W}$, where $\bar{W}$ is the mean of $e^{\beta z}$ across offspring. Thus, the population is only expected to grow (as opposed to simply being maintained by immigration) if $\bar{W}>1$. We focus on the case where the source population has $\bar{z}_{s}<0$, so that some form of selection is required for the trait to become positive in the new population, allowing it to grow.

It would be inefficient to simulate this model directly, by forming a large number of offspring, and culling them in proportion to their individual fitness. Instead, the average fitness of offspring from each possible pair of parents, $w_{i j}$, is calculated. Denoting the parental trait values by $z_{i}, z_{j}$ respectively, with exponential directional selection, $e^{\beta z}$,

$$
w_{i j}=\mathbb{E}\left[e^{\beta z}\right]=\exp \left(\beta \frac{\left(z_{i}+z_{j}\right)}{2}+\frac{\beta^{2}}{2} v_{i j}\right) .
$$

The number of surviving offspring, $N^{*}(t+1)$, is Poisson distributed with expectation $\sum_{i, j} w_{i j} / N(t)$. For each of these, parents $i, j$ are drawn with probability proportional to $w_{i j}$. This choice is represented by a pedigree matrix $P$, with dimensions $N^{*}(t+1) \times N(t) ; P_{k i}$ gives the probability that a gene in offspring $k$ came from individual $i$ in the parental population. Under the infinitesimal model, this is not distorted by knowing the trait values of the individuals involved. Thus, each row has either two entries of $1 / 2$, if that offspring has distinct parents, or one entry of 1 , if it was produced by selfing (which has probability proportional to $1 / N$ ). If offspring $k$ has parents $i, j$, then its trait value is drawn from a normal distribution with mean $\left(z_{i}+z_{j}\right) / 2+\beta v_{i j}$, and variance $v_{i j}$. The relatedness in the new population 


$$
P\left(F+\frac{1}{2}\left(I-F_{\text {diag }}\right)\right) P^{T},
$$

where $F_{\text {diag }}$ is the diagonal matrix with entries from the diagonal of $F$. New migrants (assumed to be unrelated to these $N^{*}(t+1)$ individuals and to each other) are added, to arrive at the new generation of size $N(t+1)$. For large populations, we track close relatives (those for which $F_{i j}$ is bigger than some cutoff) using a sparse matrix and set all others to have the same 'background' relatedness. Using this approximation, a population of up to $\sim 1000$ can be simulated.

\section{Establishment of a new population}

In this section, we use simulations to explore establishment of the population in the new habitat, first considering the fate of a single migrant and then continuous immigration from the source population. In this second scenario, establishment is inevitable, but we still see two competing effects: migration introduces variance which feeds selection, but reproduction with a migrant pulls back the mean trait value among offspring towards the value in the source population, thus impeding adaptation.

\subsection{Single migrants}

We begin by considering the fate of a single migrant, of trait value $z_{0}$. In the long term, density-dependent fitness must limit population size, and stabilising selection (or some other process) must limit trait evolution. However, we assume that the population is established before either of these are significant, and so take fitness to be simply $e^{\beta z}$. In the first generation, born of a single selfed migrant, before selection, the trait values of offspring are Gaussian, with mean $z_{0}$ and variance $V$. The expected number to survive selection is $\mathbb{E}\left[e^{\beta z}\right]=e^{\beta z_{0}+\beta^{2} V / 2}$. The relatedness between any two genes (sampled from either the same or distinct offspring) is $F_{i j}=1 / 2$. While the population size is small, inbreeding rapidly reduces the genetic variance, and so if the population is to survive, individuals with sufficiently large $z$ must be produced in the first few generations. The probability that this will happen depends only on the two dimensionless quantities $z_{0} / \sqrt{2 V}$ and on $\beta \sqrt{2 V}$; the latter is the standard deviation in log fitness (measured in the new habitat) of the source population. It is convenient to scale both the trait value and the fitness gradient relative to $\sqrt{2 V}$ in this way and thus set the variance in the source population to be $2 V=1$. 

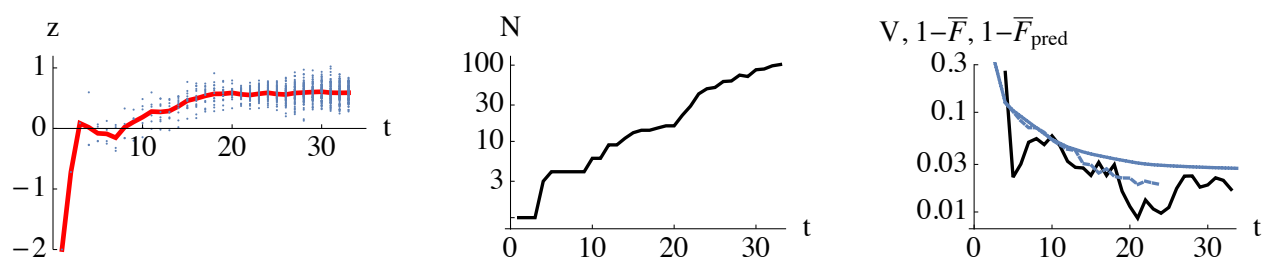

Figure 1: An example of successful establishment, starting with a single migrant with $z_{0}=-2, \beta=0.25$. Left: Values of each individual though time; thick red line shows the population mean. Middle: Population size, $N$. Right: The variance, $2 V$, and heterozygosity, $1-\bar{F}$ (solid, lower dashed), through time. The upper dashed curve shows the predicted heterozygosity, based on the population size: $\prod_{t}\left(1-\frac{1}{2 N_{t}}\right)$.

Figure 1 shows one example of a successful establishment, where we define establishment to mean reaching population size $N=100$. We start with a single migrant of value $z_{0}=-2$, and fitness gradient $\beta=0.25$; thus, recalling that $2 V=1$, the expected number of surviving offspring from this founder individual is $\mathbb{E}\left[e^{\beta z}\right]=e^{\beta z_{0}+\beta^{2} / 4}=0.62$. The population mean increases rapidly in the first few generations, as does the population size. After around 10 generations, the population mean becomes positive, after which population size rises more rapidly (Fig. 1, middle). The population increases for two reasons: first, the increase in trait mean, and hence fitness, and second, the stochastic acceleration - the expected numbers conditional on fixation must rise faster than the unconditional expectation. Because there are only a few individuals during the first few generations, the heterozygosity, $1-\bar{F}$, decreases rapidly, to only $\sim 0.05$ by the time the population is large. The actual trait variance fluctuates below this underlying heterozygosity (compare lower dashed with solid curve in Fig. 1, right). In a neutral model, one expects the average heterozygosity to decrease by a factor $1-\frac{1}{2 N}$ in each generation (upper dotted curve in Fig. 1, right); in this example the actual heterozygosity is slightly lower than this prediction. This is to be expected as selection will distort the pedigree, as fitter individuals are more likely to reproduce, and so the probability of identity between surviving offspring should exceed the neutral prediction. Based on replicate trials (Fig. 3), the probability of establishment with $z_{0}=-2, \beta=0.25$ is 0.00012. Individual replicates are highly variable.

Figure 2 shows average features of 50 successful establishments, again with $z_{0}=-2$, and for $\beta=0.125,1$ (left, right). When the population succesfully establishes, it increases rapidly, over the first $\sim 20$ generations for $\beta=0.125$, and the first $\sim 10$ generations for $\beta=1$ (top row). After this 
initial phase, the mean appears to settle to around 50; however, this is an artefact of the definition of establishment at $N \geq 100$. Once the population is above $\sim 20$, because of the variation in trait values across the population, it may rapidly fix, or it may fluctuate for 100 generations or more before taking off. As a result, the size of populations that have not yet reached $N=100$, but will eventually do so, is roughly uniformly distributed between a small number and 100.

The middle row of Fig. 2 shows the trait mean; to make runs that reached $N \geq 100$ at different times more comparable, this is plotted against population size. Offspring of successful migrants tend to have substantially higher $z$ than the parental value of $z_{0}=-2$ (averaging $\sim-1$ for $\beta=0.125$, and $\sim 0$ for $\beta=1$, compared to an average over the source population of -2 ). On average, the trait mean increases to $\sim 0.5$ by the time the population is large, though this is highly variable: some populations establish with a trait mean that is close to zero. Once populations are large, the trait mean is almost always positive, so that there is a sharp lower bound to its distribution.

The bottom row of Fig. 2 shows the genetic variance, which decreases to an average of $\sim 0.02$ for $\beta=0.125$, and $\sim 0.1$ for $\beta=1$; more variance remains when the fitness gradient is steep, because then, establishment is faster. However, the variance remaining in large populations is highly variable between runs. We set the variance in the source population to be $2 \mathrm{~V}=1$ and compare the observed variance during establishment to $2 V(1-\bar{F})$. The mean heterozygosity, $1-\bar{F}$, which determines the segregation variance, has a narrower distribution than the population variance, but almost the same mean. On average, the heterozygosity predicted from the population size, $\prod_{t}\left(1-\frac{1}{2 N_{t}}\right)$, is very close to that of the variance (compare dashed with solid lines).

Figure 3 disentangles the contributions of the chance pick of a fit individual from the source population as migrant, and the subsequent selection on its offspring, on the probability of establishment. In the left hand panel, the solid lines show the probability of establishment from a migrant sampled from a source population with mean trait value $\bar{z}_{s}$ for different fitness gradients $\beta$. (Recall that both are scaled relative to the variance $2 \mathrm{~V}$ in the source population, which is therefore set to 1 here, and that establishment is defined as reaching 100 individuals.) As $\beta$ decreases, the dependence on $\bar{z}_{s}$ becomes weaker. Indeed, in the limit $\beta=0$, we have a critical branching process in which each individual produces a Poisson number of offspring with mean 1 , and the probability of reaching 100 is $\sim 1 / 100$ independent of the initial trait value.

A migrant will typically have trait value within one standard deviation of 

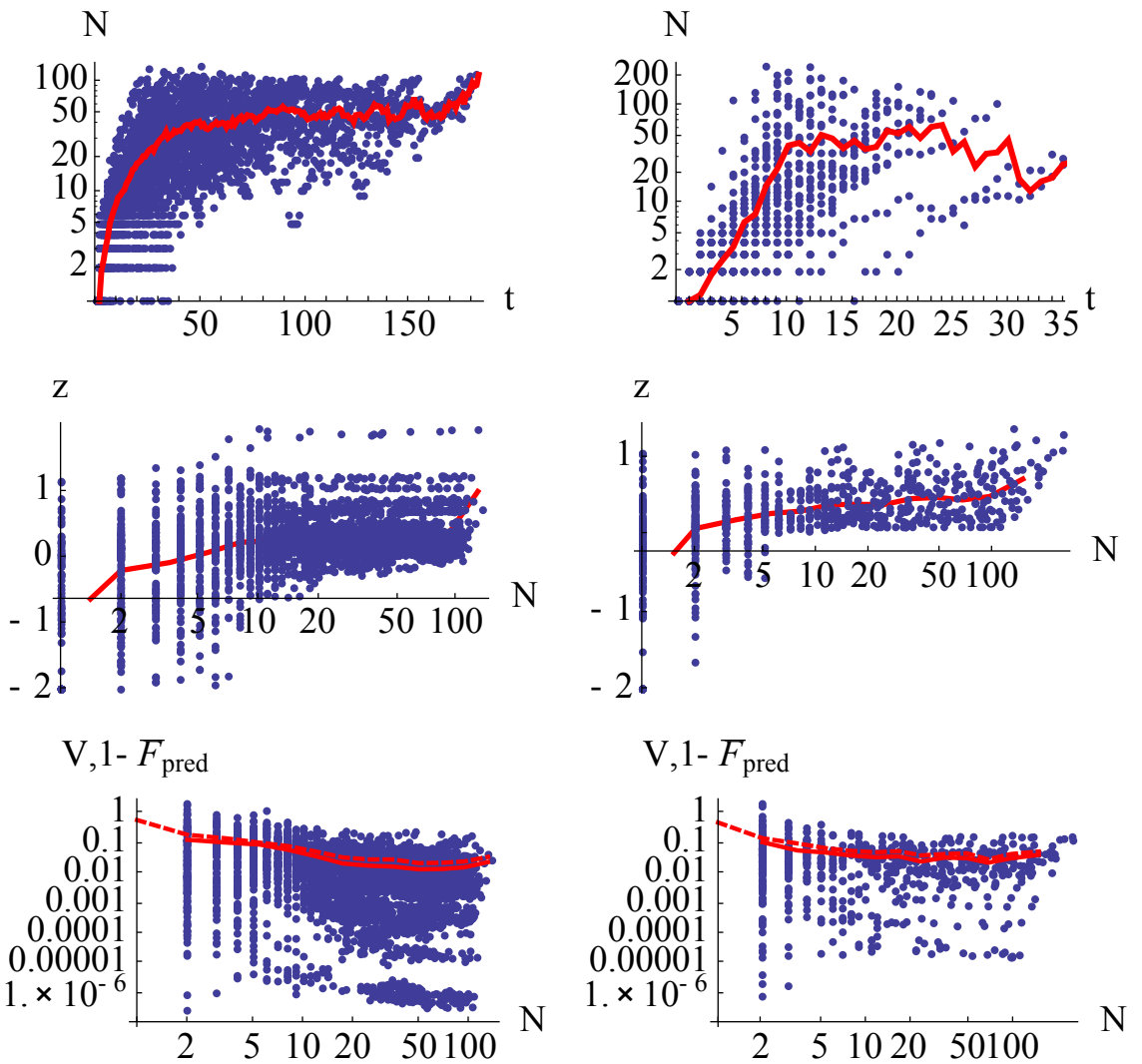

Figure 2: Population size, trait mean, and variance, for 50 successful establishments; $z_{0}=-2, \beta=0.125$ (left column), 1 (right column). (Results for $\beta=2$ were not shown, since for those only 27 runs established within $10^{5}$ trials). In each plot, the thick line shows the mean, whilst points show individual runs. Top: Population size, plotted against time. Middle: Trait mean, plotted against population size. Bottom: Trait variance, Var, plotted against population size. The mean of the variance across runs is shown by the thick line, and the mean heterozygosity predicted from population size, $1-\bar{F}_{\text {pred }}$, by the dashed line. 

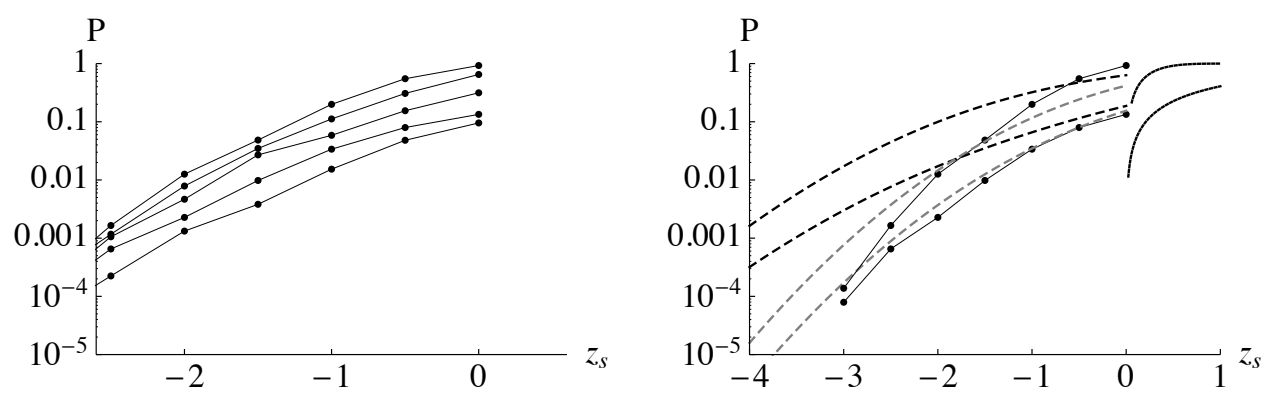

Figure 3: Left: Probability $\mathbf{P}$ of reaching population size at least $N=100$, starting from a single migrant drawn from a population with mean trait value $\bar{z}_{s}$ and variance $2 \mathrm{~V}=1$; $\beta=0.125,0.25,0.5,1,2$ (bottom to top). Right: For $\beta=0.25,2$, the leftmost solid curves show the probability of reaching at least $N=100$ for a single migrant of trait value exactly $z_{0}=\bar{z}_{s}$. The solid curves on the far right show the probability of establishment of a homozygous individual of value $z_{0}=\bar{z}_{s}$; this is the probability of establishment of a GaltonWatson branching process with Poisson offspring distribution with mean $e^{\beta \bar{z}_{s}}$ (which we have approximated by the survival probability). This is zero for $\bar{z}_{s}<0$. The black dashed curves show the probability of establishment of a migrant chosen randomly from a source population with mean $\bar{z}_{s}$ and variance $2 V=1$. Grey dashed curves show the same, but for a homozygous individual, so that the new population cannot evolve. The dashed curves are calculated by integration over the values shown by the solid curves. Estimates are based on the number of trials required to successfully establish 50 populations. Each of the 50 runs made a maximum of $10^{5}$ trials; values here are maximum likelihood estimates of the establishment probability, assuming an exponential distribution for time to establishment, truncated at $10^{5}$. 
$\bar{z}_{s}$, but selection acting on the variance among the migrant's offspring means that the descendants of a migrant which itself is not expected to grow, can still have a positive chance of establishment.

The solid lines in the right hand plot of Fig. 3 show the probability of establishment when the migrant trait is exactly $z_{0}=\bar{z}_{s}$. The black dashed curves in this panel allow direct comparison with the probability of establishment from a randomly chosen individual from the source population with mean trait value $\bar{z}_{s}$ and variance $2 V=1$ for $\beta=0.125,2$. Because the migrant may be a few standard deviations above the mean, for negative $\bar{z}_{s}$ these probabilities are much higher than for a migrant whose trait is exactly $z_{0}=\bar{z}_{s}$.

The contribution of selection on the variance among offspring (as opposed to on the fitness of the original migrant) can be seen by comparison with the probability of establishment from a homozygous individual, whose offspring necessarily have the same trait value as the parent. This is just the establishment probability of a Galton-Watson branching process with Poisson offspring distribution with mean $e^{\beta z_{0}}$. We approximate this by the survival probability (recalling that establishment means reaching $N=100$ ). It is zero for $z_{0}<0$ and increases steeply with $\beta z_{0}$ (curves on the far right). Comparing the curves for large positive values of $z_{0}$, we see that selection on variance has little effect on the probability of establishment if the initial migrant is already well adapted.

The grey dashed lines in the right hand panel of Fig. 3 show the probability of establishment from a homozygous individual drawn from a population with trait mean $\bar{z}_{s}$ and variance $2 V=1$. Comparing with the black dashed lines, we see that although genetic variance is rapidly lost during the first few generations, selection during this time can greatly increase the chances of successful establishment. Indeed, even after the population has become large, some genetic variation remains, and so further adaptation will occur even without new mutation.

What we conclude from Fig. 3 is that establishment is feasible even when $\bar{z}_{s}$ is 3 or 4 genetic standard deviations below the threshold $z=0$ for growth, and when an individual with trait value given by the mean in the source population would be extremely unfit in the new habitat. This is quantified through some numerical examples in Table 1. Roughly speaking, the change in trait value required for the population to increase is contributed to a similar degree by the chance migration of an individual that is unusually well adapted to the new habitat, and by the subsequent evolution of the new population. 
Table 1: Threshold values for establishment probabilities of $10^{-4}$. (i) Top panel, $z_{0}^{c}$, the critical value of $z_{0}$ above which establishment probability is greater than $10^{-4}$; (ii) Middle panel, $\bar{z}_{s}^{c}$, the critical value of $\bar{z}_{s}$ for which establishment from a migrant picked at random from the source population has probability $10^{-4}$; (iii) Bottom panel, $\widetilde{z}_{s}^{c}$, the same as (ii) except that the migrant is taken to be homozygous. We have normalised so that $2 V=1$.

(i)

\begin{tabular}{lll}
$\beta$ & $z_{0}^{c}$ & $\exp \left(\beta z_{0}^{c}\right)$ \\
\hline 0.125 & -2.01 & 0.78 \\
0.25 & -2.04 & 0.60 \\
0.5 & -2.00 & 0.37 \\
1 & -1.84 & 0.16 \\
2 & -1.62 & 0.04
\end{tabular}

(ii)

\begin{tabular}{lll}
$\beta$ & $\bar{z}_{s}^{c}$ & $\exp \left(\beta \bar{z}_{s}^{c}\right)$ \\
\hline 0.125 & -3.64 & 0.63 \\
0.25 & -3.82 & 0.39 \\
0.5 & -3.90 & 0.14 \\
1 & -3.92 & 0.02 \\
2 & -3.99 & 0.00
\end{tabular}

(iii)

\begin{tabular}{lll}
$\beta$ & $\widetilde{z}_{s}^{c}$ & $\exp \left(\beta \widetilde{z}_{s}^{c}\right)$ \\
\hline 0.125 & -2.97 & 0.69 \\
0.25 & -3.15 & 0.45 \\
0.5 & -3.31 & 0.19 \\
1 & -3.45 & 0.03 \\
2 & -3.55 & 0.00
\end{tabular}




\subsection{Steady migration}

Now, suppose that there is a steady influx of migrants from the source population. We still neglect density-dependence and stabilising selection, and so establishment is inevitable: eventually, a large enough population will be established, with sufficient genetic variation, to allow adaptation and growth to indefinitely high numbers. When the influx is low, establishment depends on single migrants, and can be predicted from the results of the previous section (dashed lines at left of Fig. 4). However, as the migration rate increases, interactions between migrants become important, in two opposing ways. First, migrants introduce genetic variation, which can greatly increase the efficiency of selection. However, migration also pulls the trait mean back towards the value in the source population, which impedes adaptation. There is a strong positive feedback here, because small populations are especially liable to the swamping effects of gene flow, which impedes their adaptation to local conditions.

Figure 4 shows how the expected time to establishment (defined as reaching $N \geq 100$ ) depends on the number of migrants per generation, $M$, for $\beta \sqrt{2 V}=0.25$. Overall, we see that for $z_{s} / \sqrt{2 V}>-1.5$ (lowest two solid curves), the mean time to establishment decreases with immigration, and becomes very fast for large $M$. However, for higher levels of divergence $\left(z_{s} / \sqrt{2 V} \leq-1.5\right)$ there is an intermediate migration rate, $M$, which minimises the time to establishment; for large migration rates, establishment takes an extremely long time.

\section{Approximating the dynamics of escape}

While the population is rare in the new environment, we can neglect density dependent regulation. Conditional on the trait values, each possible pair of individuals in the population, independently, leaves behind a random number of offspring, with a distribution that depends on the parental traits. This is highly reminiscent of the nonlinear stochastic growth models introduced in a series of papers beginning with Kesten (1970); see Kesten (1971) for a review. Kesten's model is one of a diploid population and incorporates multiple types, and so in particular could be used to model multilocus traits. However, the only really satisfying analytic results currently available are when the type of an offspring is that of one of its parents. In our setting, in which the variance released by recombination plays a crucial role, these results do not apply. Here we shall satisfy ourselves with some simple caricatures of the joint evolution of population size and trait distribution, deferring a study of more sophisticated models to future work. 


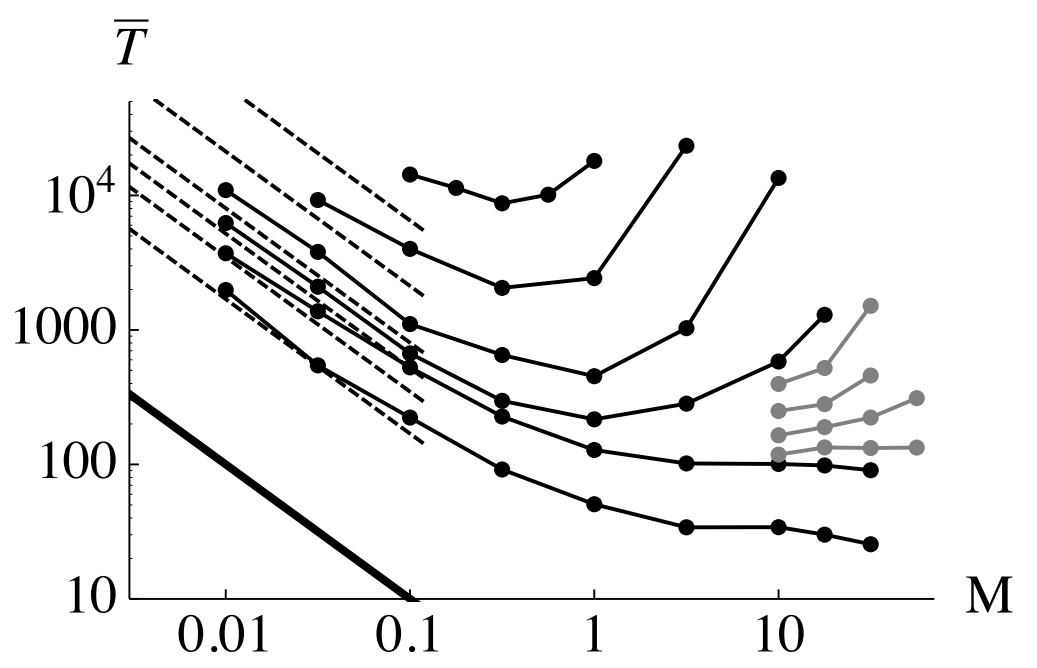

Figure 4: Mean time to establishment, for $\beta=0.25$, given migration at rate $M$ from a source population with mean $\bar{z}_{s}$ and variance $2 V=1 ; \bar{z}_{s}=-1,-1.5,-2,-2.5,-3.5$ (bottom to top, black lines). Additional values, $\bar{z}_{s}=-1.55,-1.6,-1.65,-1.7$ are shown in grey, for large $M$ (right of figure). The dashed lines at the left give the prediction, $\bar{T}=\frac{1}{M P}$, from the probability of establishment, $P$, of a single migrant, drawn randomly from the source population (Fig. 3, right, dashed curves). The thick line at lower left shows the lower bound, $\bar{T}=1 / M$, which would obtain if the first migrant to arrive immediately established a new population. Establishment is defined as a population of $N \geq 100$ for $M<10$, and $N \geq 300$ for $M>10$. Estimates of $\bar{T}$ are based on 100 replicates for each $\bar{z}_{s}, M$. Relatedness, $F$, is tracked between each pair of individuals up to $N=50$, above which it is replaced by the mean relatedness. 


\section{1. 'Deterministic' approximations}

To understand why establishment becomes difficult at high migration rates when divergence is above a threshold value, we shall make the approximation that the trait distribution across the whole population is Gaussian. As a first approximation, we shall further suppose that the population size and the trait mean and variance evolve deterministically. We shall refer to this as the deterministic approximation. We emphasize that the Gaussian distribution across the population is not a consequence of using the infinitesimal model, which, in the presence of selection, only guarantees that within each family the trait distribution is normally distributed.

We expect this to be a reasonable approximation if migration rates are moderately high and the selection gradient is not too extreme (c.f. Tufto 2000). Under the infinitesimal model, each diploid migrant carries half of the genetic variance in the source population, and so modest rates of migration into a small 'sink' population can maintain high genetic variance, without causing large deviations from a Gaussian.

Tufto (2001) examines a similar deterministic model, assuming a fixed genetic variance and stabilising selection. He is primarily concerned with identifying when migration can reduce population size by introducing maladapted individuals, but notes that the positive feedback between gene flow, adaptation, and population size can lead to multiple stable equilibria. Ronce and Kirkpatrick (2001) also identify multiple equilibria for a model of exchange between two demes with different optima under stabilising selection.

In Appendix A, we develop a recursion for the joint evolution of population size, trait mean, trait variance and average identity, which fits the dynamics well, and predicts a critical divergence, beyond which maladaptation becomes stable. Here, we develop a simpler recursion, by assuming that the variance remains approximately constant, despite selection and migration, and assuming that the identity is zero. In the more sophisticated models that follow, we shall routinely use the notation $V^{*}=V\left(1-F_{*}\right)$ for the variance (before selection) among offspring of two individuals from the new population, where $V$ is the segregation variance among offspring of two unrelated individuals and $F_{*}$ is the average identity in the new population. We shall also use that notation here, even though we are assuming $F_{*}=0$. Under the infinitesimal model, directional selection does not change the variance, and so if the variance across the population is approximately constant it will be $2 V^{*}$.

Our aim is to write down deterministic equations which, under these assumptions, govern the joint evolution of the expected population size and 
the mean value of the trait across the population. With a slight abuse of notation, we denote these quantities by $N(t), \bar{z}(t)$, respectively. Since the within family variance is $V^{*}$, the mean fitness, $\bar{W}$, among offspring is

$$
\bar{W}=\mathbb{E}\left[\exp \left(\beta \frac{\left(Z_{1}+Z_{2}\right)}{2}+\frac{\beta^{2} V^{*}}{2}\right)\right],
$$

where $Z_{1}$ and $Z_{2}$ are the traits of two parents sampled at random from the population. (Here we are using our assumption that identity is zero in both the new and the source populations, so that the within family variance is the same irrespective of whether one or both of the parents is a newly arrived migrant or not, but see Appendix A for the more general case.) The distribution of traits across the population is Gaussian with mean $\bar{z}$ and variance $2 V^{*}$, giving

$$
\bar{W}=\exp \left(\beta \bar{z}+\beta^{2} V^{*}\right),
$$

and so after reproduction and the subsequent migration,

$$
N(t+1)=M+N(t) \exp \left(\beta \bar{z}(t)+\beta^{2} V^{*}\right) .
$$

The mean trait among these individuals is

$$
\bar{z}(t+1)=\frac{1}{N(t+1)}\left(M \bar{z}_{s}+N(t) \mathbb{E}\left[z e^{\beta z}\right]\right),
$$

where the expectation is with respect to the distribution of the trait among offspring (before selection) and can be calculated by differentiating $\bar{W}$ with respect to $\beta$. This yields

$$
\begin{aligned}
\bar{z}(t+1) & =\frac{1}{N(t+1)}\left(M \bar{z}_{s}+N(t)\left(\bar{z}(t)+2 \beta V^{*}\right) e^{\beta \bar{z}(t)+\beta^{2} V^{*}}\right) \\
& =\left(\bar{z}(t)+2 \beta V^{*}\right)\left(1-\frac{M}{N(t+1)}\right)+\bar{z}_{s} \frac{M}{N(t+1)} \\
& =\bar{z}(t)+2 \beta V^{*}\left(1-\frac{M}{N(t+1)}\right)-\frac{M}{N(t+1)}\left(\bar{z}(t)-\bar{z}_{s}\right) .
\end{aligned}
$$

It is convenient to rescale equations (4) and (5) by setting $n=N / M, \alpha=$ $\beta \sqrt{2 V^{*}}$ and $y=\left(\bar{z}-\bar{z}_{s}\right) / \sqrt{2 V^{*}}$. Then the equations become

$$
n(t+1)=1+n(t) W_{s} e^{\alpha y(t)}, \quad y(t+1)=(y(t)+\alpha)\left(1-\frac{1}{n(t+1)}\right),
$$


where

$$
W_{s}=\exp \left(\beta \overline{z_{s}}+\beta^{2} V^{*}\right)
$$

is the mean growth rate of the source population in the new conditions.

If $W_{s}$ is above some critical value, $W_{s, \text { crit }}$, population size and trait increase together even from low values, regardless of $M$. For smaller values of $W_{s}$, there are two equilibria, one stable and one unstable. In this case, the population may be unable to grow, regardless of how large is $M$; instead, it is maintained by migration as a poorly adapted 'sink'. Although an increase in $M$ increases the population size, it also impedes adaptation, and therefore prevents indefinite growth. The critical value can be seen at the right of Fig. 4 (grey lines): for large $M$, where the system approaches the deterministic regime, there is a critical value of $\bar{z}_{s} \sim 1.57$, above which the expected time to establishment becomes indefinitely large.

To find the critical value, observe that, from (6), at equilibrium $y(t)=$ $y(t+1)=\alpha(n(t+1)-1)$, i.e., $y_{\text {crit }}=\alpha\left(n_{\text {crit }}-1\right)$ and writing $f(n)=$ $1+n W_{s} e^{\alpha^{2}(n-1)}$ we must solve

$$
n=f(n), \quad 1=f^{\prime}(n) .
$$

This yields a quadratic in $n$ whose positive solution is

$$
n_{\text {crit }}=\frac{\alpha^{2}+\sqrt{\alpha^{4}+4 \alpha^{2}}}{2 \alpha^{2}}=\frac{1}{2}\left(1+\sqrt{1+4 / \alpha^{2}}\right) .
$$

In our original variables, this becomes

$$
N_{\text {crit }}=\frac{M}{2}\left(1+\sqrt{1+2 /\left(\beta^{2} V^{*}\right)}\right),
$$

and substituting in (7) to find $W_{s, \text { crit }}$ we find

$$
W_{s, \text { crit }}=\frac{n_{\text {crit }}-1}{n_{\text {crit }}} e^{-\alpha^{2}\left(n_{\text {crit }}-1\right)}=\left(1-\frac{M}{N_{\text {crit }}}\right) e^{-\alpha^{2}\left(N_{\text {crit }}-M\right) / M},
$$

and finally, since $W_{s, \text { crit }}=\exp \left(\beta \bar{z}_{s, \text { crit }}+\beta^{2} V^{*}\right)$, we find

$$
\beta \bar{z}_{s, \text { crit }}=-\frac{1}{2} \alpha\left(\sqrt{4+\alpha^{2}}\right)-\log \left(\frac{\alpha+\sqrt{4+\alpha^{2}}}{-\alpha+\sqrt{4+\alpha^{2}}}\right) .
$$

For $\alpha=\beta \sqrt{2 V^{*}} \ll 1, \beta \bar{z}_{s, \text { crit }} \sim-2 \alpha$. For $\alpha \gg 1, \beta \bar{z}_{s, \text { crit }} \approx-\alpha^{2} / 2-2 \log \alpha$.

The solid line in Fig. 5 (top) shows the critical log fitness of an average 
migrant above which establishment is inevitable in this deterministic limit, $\beta \bar{z}_{s, \text { crit }}$, plotted against the standard deviation of log fitness in the source population, $\alpha=\beta \sqrt{2 V^{*}}$. The dashed line shows the more sophisticated approximation of Appendix A, which takes into account the inbreeding in the new population and the different within-family variances of offspring resulting from different combinations of native and migrant parents. The lower plot shows the corresponding critical fitness of the source population in the new environment, $W_{s, \text { crit }}$.

\subsection{The diffusion approximation to the stochastic dynamics}

In this section we shall continue to assume that migration rates are moderately high so that the trait distribution across the population has (approximately) a Gaussian distribution, but we extend the analysis of the last section to incorporate stochastic fluctuations in the population size and trait mean to capture demographic stochasticity and random sampling drift. We shall use a continuous time approximation.

The first step is to find the appropriate continuous time approximation to the discrete equations (4), (5). If $\beta \bar{z}+\beta^{2} V^{*}$ is small, neglecting the second order term $\beta^{2} V^{*}$, we arrive at the pair of ordinary differential equations:

$$
\begin{aligned}
\frac{d N(t)}{d t} & =M+\beta \bar{z}(t) N(t) \\
\frac{d \bar{z}(t)}{d t} & =2 \beta V^{*}\left(1-\frac{M}{2 N(t)}\right)-\frac{M}{N(t)}\left(\bar{z}(t)-\bar{z}_{s}\right) .
\end{aligned}
$$

In fact, as we explain in Appendix B, if we wanted to accumulate a smaller error (of order at most $\beta^{2}(M+N)$ per generation) in using this differential equation for population size in place of our discrete recursion, we should (at the expense of much more complex expressions in what follows) replace the immigration rate $M$ in the first equation by $M(1-\beta \bar{z}(t) / 2)$. With this equation the error is order $\beta \bar{z} M+\beta^{2}(M+N)$. Since our model is in any case only a caricature, we take these simpler equations.

We now add random perturbations $\zeta_{N}, \zeta_{\bar{z}}$ to these equations. To see what form these should take, first recall that the variance of the trait across the population is taken to be $2 V^{*}$. If we take a random sample of size $N$ from a population with mean $\bar{z}$ and variance $2 V^{*}$, then the mean trait value across the sample will have mean $\bar{z}$ and variance $2 V^{*} / N$. The perturbation $\zeta_{\bar{z}}$ should therefore have mean zero and variance per unit time (corresponding to a single generation) $2 V^{*} / N$. In the notation of, for example, Gardiner (2004), we write $\left\langle\zeta_{\bar{z}}^{2}\right\rangle=2 V^{*} / N$. 

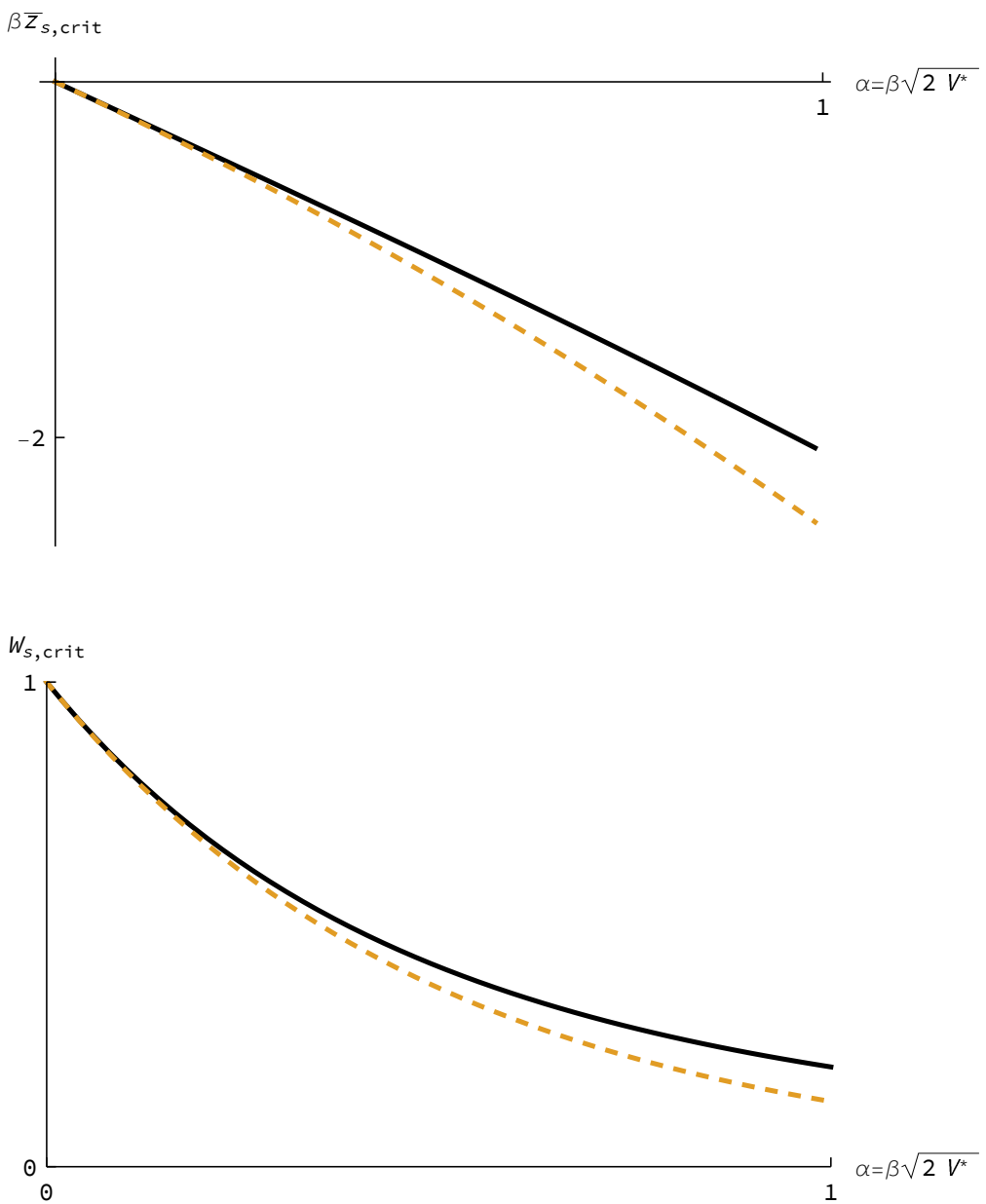

Figure 5: Top: The critical log fitness of an average migrant, $\beta \bar{z}_{s, \text { crit }}$, above which establishment is inevitable in the deterministic limit, plotted against the standard deviation of $\log$ fitness in the source population, $\beta \sqrt{2 V^{*}}$. The solid line assumes constant variance $2 V^{*}$ (Eq. 9). The dashed line allows variance to evolve (Appendix A). Bottom: The critical fitness of an average migrant in the new environment, $W_{s, \text { crit }}=\exp \left(\beta \bar{z}_{s, \text { crit }}+\beta^{2} V^{*}\right)$. 
For the perturbation $\zeta_{N}$, we appeal to Feller (1951). For a large population, Feller showed that one can approximate a branching process by a diffusion in which fluctuations are of order $\sqrt{N}$. Again using the notation of Gardiner (2004), we write $\left\langle\zeta_{N}^{2}\right\rangle=N$.

The dynamics can be described by a potential, $U$, given by

$$
U=M \log N+\beta\left(N-\frac{M}{2}\right) \bar{z}-\frac{M}{4 V^{*}}\left(\bar{z}-\bar{z}_{s}\right)^{2},
$$

in terms of which we have

$$
\begin{array}{ll}
\frac{d N}{d t}=N \frac{\partial U}{\partial N}+\zeta_{N}=M+\beta \bar{z} N+\zeta_{N}, & <\zeta_{N}^{2}>=N ; \\
\frac{d \bar{z}}{d t}=\frac{2 V^{*}}{N} \frac{\partial U}{\partial \bar{z}}+\zeta_{\bar{z}}=2 \beta V^{*}\left(1-\frac{M}{2 N}\right)-\frac{M}{N}\left(\bar{z}-\bar{z}_{s}\right)+\zeta_{\bar{z}}, & <\zeta_{\bar{z}}^{2}>=\frac{2 V^{*}}{N} .
\end{array}
$$

Equivalently, we could write the equations for $N$ and $\bar{z}$ as a pair of stochastic differential equations

$$
\begin{aligned}
d N(t) & =(M+\beta \bar{z}(t) N(t)) d t+\sqrt{N(t)} d B_{t}^{1}, \\
d \bar{z}(t) & =\left(2 \beta V^{*}\left(1-\frac{M}{2 N(t)}\right)-\frac{M}{N(t)}\left(\bar{z}(t)-\bar{z}_{s}\right)\right) d t+\sqrt{\frac{2 V^{*}}{N(t)}} d B_{t}^{2},
\end{aligned}
$$

where $B^{1}$ and $B^{2}$ are independent Brownian motions.

If there were a stationary distribution with density $\psi(N, \bar{z})$, then, see e.g. Gardiner (2004), using $\propto$ to denote 'is proportional to', $\S 5.3 .3$, it would satisfy

$$
\psi(N, \bar{z}) \propto \frac{e^{2 U}}{N}=N^{2 M-1} \exp \left(\beta(2 N-M) \bar{z}-\frac{M}{2 V^{*}}\left(\bar{z}-\bar{z}_{s}\right)^{2}\right) .
$$

This expression diverges for large $N, \bar{z}$, reflecting the fact that our model doesn't prevent fitness and population size from growing without bound, but if we modify our model to include regulation of these quantities, $\psi(N, \bar{z})$ should approximate the density of the population while it is near to a stable 'sink' equilibrium, when that exists. In the next section, we extend it to include density regulation and stabilising selection.

Equation (10) has a simple form, with three terms that correspond to migration that increases population size $\left(N^{2 M-1}\right)$, directional selection on the trait $\left(e^{\beta(2 N-M) \bar{z}}\right)$, and gene flow that pulls the trait mean towards the 

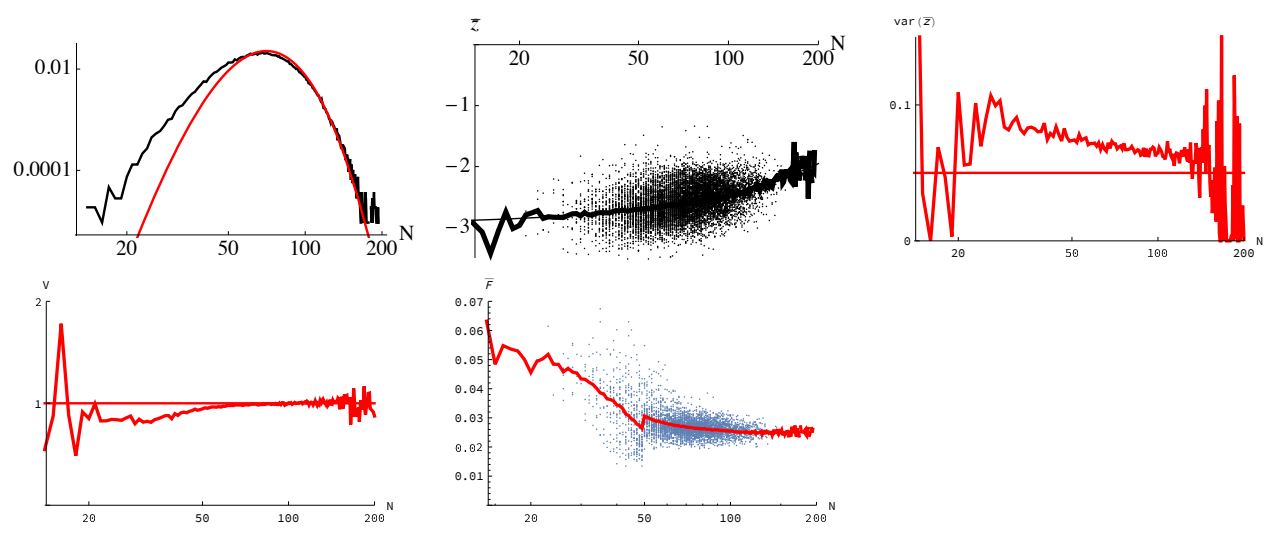

Figure 6: The distribution of $N, \bar{z}$ for a population trapped in a sink; $z_{s}=-3, \beta=$ $0.05, M=10$. 11 replicate simulations were made with a total of $10^{5}$ generations; the first 200 generations were discarded. Top left: the distribution of $N$, compared with that predicted by Eq. (11), $N^{2 M-1} \exp \left((2 N-M) \beta \bar{z}_{s}+(2 N-M)^{2} \beta^{2} / 4 M\right)$ (red). Top middle: The trait mean, conditional on $N$ compared with the predicted $\bar{z}_{s}+\beta V^{*}(2 N-$ $M) / M$ ), assuming $2 V^{*}=1$. Top right: The variance of the mean compared with the predicted $\operatorname{var}(\bar{z})=1 / 2 M$. Bottom left: The population variance, $2 V^{*}$, conditional on $N$, compared with the assumed $2 V^{*}=1$. Bottom middle: Mean identity, $\bar{F}$, conditional on $N$.

source $\left(e^{-M\left(\bar{z}-z_{s}\right)^{2} / 2 V^{*}}\right)$.

For given $N$, the trait mean is normally distributed, with variance $V^{*} / M$, and mean $\mathbb{E}[\bar{z}]=\bar{z}_{s}+\beta V^{*}(2 N-M) / M$; this is the deterministic equilibrium in which selection $2 \beta V^{*}(1-M / 2 N)$ increases the trait mean, but is opposed by gene flow at rate $M / N$. By 'completing the square' and writing the exponent in (10) as a term of the form $A(\bar{z}-B)^{2}$ plus a remainder that is independent of $\bar{z}$, we can integrate over $\bar{z}$ to recover the marginal distribution $\psi(N)$ of $N$, which we find satisfies

$$
\psi(N) \propto N^{2 M-1} \exp \left(\beta^{2}(2 N-M)^{2} \frac{V^{*}}{2 M}+\beta(2 N-M) \bar{z}_{s}\right) .
$$

If $M>1 / 2$ (which is required for genetic variance, $2 V^{*}$, to be high and approximately constant), and if $\bar{z}_{s}<-2 \sqrt{V^{*}(1-1 /(2 M))}+\beta V^{*} / 2 \sim-2 \sqrt{V^{*}}$, then the distribution has a peak at low density, and with $\bar{z}<0$, which represents a metastable 'sink', in which the population is maintained by gene flow despite its maladaptation. Figure 6 compares this approximation with the distribution of $\bar{z}, N$ for $M=10$. 


\section{Understanding the new equilibrium}

\subsection{Equilibrium of deterministic model}

Once the new population is established, it will grow and adapt. Eventually, its size must be limited by some form of density dependence, and the trait will reach some equilibrium mean and variance. Despite inbreeding, there will be some residual genetic variance (the amount being highly variable), which will slowly be augmented by new mutation. We will first outline how trait evolution may be limited, and then develop a diffusion approximation for the full process. In the following section, we complete the analysis by using the deterministic approximation to estimate the degree to which the newly established population is reproductively isolated from its progenitor.

Suppose that fitness decreases linearly with population density, at rate $\gamma$, giving a continuous-time approximation:

$$
\begin{aligned}
& \frac{d N(t)}{d t}=M+N(t) \beta \bar{z}(t)-\gamma N(t)^{2} \\
& \frac{d \bar{z}(t)}{d t}=2 \beta V^{*}\left(1-\frac{M}{2 N(t)}\right)-\frac{M}{N(t)}\left(\bar{z}(t)-\bar{z}_{s}\right) .
\end{aligned}
$$

A homogeneous population with trait $\bar{z}$ would equilibrate at

$$
N=\frac{1}{2 \gamma}\left(\beta \bar{z}+\sqrt{\beta^{2} \bar{z}^{2}+4 M \gamma}\right)
$$

but the trait is also evolving. For the trait mean to be in equilibrium, we must have $\bar{z}=\bar{z}_{s}+2 \beta V^{*} \frac{2 N-M}{M}$, which implies equilibria for population size at:

$$
N=\frac{M}{2\left(2 \beta^{2} V^{*}-M \gamma\right)}\left(-\beta \bar{z}_{s}+\beta^{2} V^{*} \pm \sqrt{\left(\beta \bar{z}_{s}-\beta^{2} V^{*}\right)^{2}-8 \beta^{2} V^{*}+4 M \gamma}\right) .
$$

There are three distinct regimes. If $2 \beta^{2} V^{*}<\gamma M$, the trait cannot evolve fast enough to overcome migration and density regulation, and the population will be trapped in a unique 'sink' equilibrium. If

$$
8 \beta^{2} V^{*}-\left(\beta \bar{z}_{s}-\beta^{2} V^{*}\right)^{2}<4 \gamma M<8 \beta^{2} V^{*},
$$

then there is a stable 'sink', but the population can escape to indefinitely 
high density if it can get beyond an unstable saddle point. Finally, if

$$
4 \gamma M<8 \beta^{2} V^{*}-\left(\beta \bar{z}_{s}-\beta^{2} V^{*}\right)^{2},
$$

the population will expand indefinitely, even when it starts at low density and is poorly adapted.

We see that simply adding linear density-dependence to our equation for population size cannot maintain a stable equilibrium at high density, in addition to the stable 'sink' which exists for $8 \beta^{2} V^{*}-\left(\bar{z}_{s}-\beta^{2} V^{*}\right)^{2}<4 \gamma M$, because the trait mean increases indefinitely under directional selection. However, there are (at least) three ways by which the indefinite adaptive increase of the population might be limited. First, density-dependence might be steeper than linear (e.g. $\sim-\gamma N^{a}$ for $a>1$ ); then, population size would stabilise and the trait would be maintained in a migration-selection balance. Second, under purely directional selection, eventually favourable alleles would fix (and the infinitesimal model would break down), genetic variance would decrease, and mutation pressure would reduce the mean. Third, the trait might be under stabilising selection towards $z_{\text {opt }}>0$, with $\log$ fitness $\frac{s}{2}\left(z_{\mathrm{opt}}^{2}-\left(\bar{z}-z_{\mathrm{opt}}\right)^{2}\right)$, preventing its indefinite increase. We have chosen this form for log fitness so that the threshold for positive growth is at $\bar{z}=0$. Near that threshold, the trait would still be under approximately directional selection with $\beta \sim s z_{\mathrm{opt}}$, provided $z_{\mathrm{opt}} \gg \bar{z}_{s}, \sqrt{2 V^{*}}$.

\subsection{Diffusion approximation at a stationary state}

We now extend the diffusion approximation to include density-dependence and stabilising selection; it is not immediately obvious how to include mutation or fixation of favourable alleles in the quantitative genetic model in a consistent way, and so we do not consider these cases. We shall use $2 \hat{V}$ to denote the variance of the trait across the population. As we explain in Appendix C, under stabilising selection, negative linkage disequilibrium results in $2 \hat{V}<2 V^{*}$ (although for small $s, \hat{V} \sim V^{*}$ ). The potential, $U$, is modified to allow density dependence $-\gamma N^{a}$, and arbitrary mean fitness $\bar{r}$. 
The corresponding diffusion approximation takes the form

$$
\begin{aligned}
U & =M \log N+N \bar{r}-\frac{M}{4 \hat{V}}\left(\bar{z}-\bar{z}_{s}\right)^{2}-\frac{\gamma}{a} N^{a}, \\
\frac{d N}{d t} & =N \frac{\partial U}{\partial N}+\zeta_{N}=M+N \bar{r}-\gamma N^{a}+\zeta_{N}, \quad<\zeta_{N}^{2}>=N, \\
\frac{d \bar{z}}{d t} & =\frac{2 \hat{V}}{N} \frac{\partial U}{\partial \bar{z}}+\zeta_{\bar{z}}=2 \hat{V} \frac{\partial \bar{r}}{\partial \bar{z}}-\frac{M}{N}\left(\bar{z}-\bar{z}_{s}\right)+\zeta_{\bar{z}}, \quad<\zeta_{\bar{z}}^{2}>=\frac{2 \hat{V}}{N} .
\end{aligned}
$$

Hence, the stationary density $\psi(N, \bar{z})$ satisfies

$$
\psi(N, \bar{z}) \propto \frac{e^{2 U}}{N}=N^{2 M-1} \exp \left(2 N \bar{r}-\frac{M}{2 \hat{V}}\left(\bar{z}-\bar{z}_{s}\right)^{2}-\frac{2 \gamma}{a} N^{a}\right) .
$$

This is valid on the assumption that the genetic variance changes slowly, so that the population size and trait mean approach the stationary distribution conditional on the current genetic variance. Stabilising selection would be represented by

$$
\bar{r}=\frac{s}{2}\left(z_{\mathrm{opt}}^{2}-\left(\bar{z}-z_{\mathrm{opt}}\right)^{2}\right) .
$$

Then, for example, if $a=2$ and we normalise so that $2 \hat{V}=1$, the density of the stationary distribution $\psi(N, \bar{z})$ satisfies

$$
\begin{aligned}
& \psi(N, \bar{z}) \propto N^{2 M-1} \\
& \quad \times \exp \left(-M\left(\bar{z}-\bar{z}_{s}\right)^{2}-s\left(N-\frac{M}{2}\right)\left(\left(\bar{z}-z_{\mathrm{opt}}\right)^{2}-z_{\mathrm{opt}}^{2}\right)-\gamma N^{2}\right) .
\end{aligned}
$$

As before, integrating over the trait we find that the density of the marginal distribution of the population size, $\psi(N)$, satisfies

$$
\begin{aligned}
\psi(N) & \propto \frac{N^{2 M-1}}{\sqrt{M+s\left(N-\frac{M}{2}\right)}} \\
& \times \exp \left(\frac{-s M(N-M / 2)\left(z_{\mathrm{opt}}-\bar{z}_{s}\right)^{2}}{M+s\left(N-\frac{M}{2}\right)}+s\left(N-\frac{M}{2}\right) z_{\mathrm{opt}}^{2}-\gamma N^{2}\right) .
\end{aligned}
$$


Alternatively, if we condition on the population size, then the trait has mean and variance given by

$$
\mathbb{E}[z \mid N]=\frac{M \bar{z}_{s}+s z_{\mathrm{opt}}\left(N-\frac{M}{2}\right)}{M+s\left(N-\frac{M}{2}\right)}, \quad \operatorname{Var}(\bar{z} \mid N)=\frac{1}{2\left(M+s\left(N-\frac{M}{2}\right)\right)} .
$$

Figure 7 shows the result of a simulation of a population subject to stabilising selection towards an optimum at $z_{\mathrm{opt}}=5$ with $s=0.02$ and density regulation as above with $a=2$ and $\gamma=0.001$. There are two metastable states. Over a total of 55,000 generations, 8 of 11 replicate simulations, each started from a single migrant, jump from low density to high density, and none jump in the opposite direction. Thus jumps are too rare for it to be feasible to estimate the rates of transition in the two directions, and therefore the relative weight of each metastable state, but the diffusion approximation does predict the joint distribution of $N, \bar{z}$, given that the population is in one or other of the two metastable states. This prediction is shown by the red curve at top left of Fig. 7. The predicted distribution has been separated into two peaks which are then weighted by the observed weights; thus the fit is to the shape of each peak separately, not to their relative weights. The population variance is assumed to be $2 V^{*}$, the mean identity being calculated from pooled data at low and high densities $(0.29,0.23$ respectively). The other four panels of Fig. 7 show the expected mean, the variance of the trait mean, the variance within populations, and the mean identity. The expectation and the variance of the trait mean match well, except in the transition region $(20<N<150)$, where there is little data. The variance within populations is somewhat lower than the predicted $2 V^{*}$ at low density, but somewhat higher at high density (bottom left).

\section{Introgression into the new population}

Once the new population is established with large numbers, and has adapted to its new environment, it will still receive migrants from its progenitor population. As we see, for example, from (13), these will impede adaptation by pulling the trait mean back, but given that establishment was possible, the new population will be able to remain distinct unless conditions change. Moreover, introgression will be reduced both because the new population is larger, and because it is better adapted. We shall suppose that the established population is sufficiently large (or migration rates are sufficiently small) that $M \ll N$. Here, we calculate the reproductive value of an individual backcrossing into such a population - that is its expected 


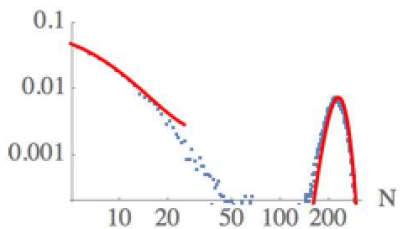

$\begin{array}{llll}10 & 20 & 50 & 100200\end{array}$

variance

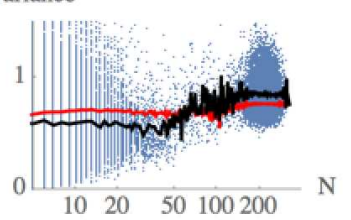

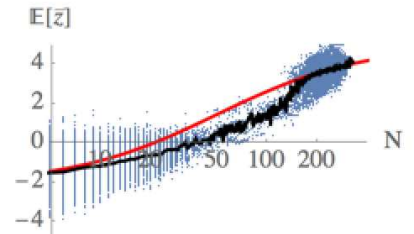

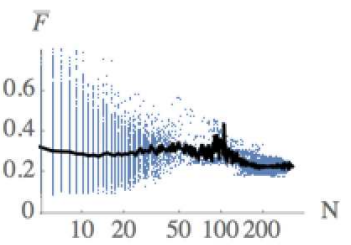

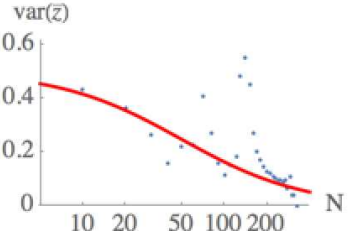

102050100200

Figure 7: The distribution of $N, \bar{z}$ for a population subject to stabilising selection, $s=0.02$, towards an optimum at $z_{\mathrm{opt}}=5$, and density regulation with $a=2, \gamma=0.001$; migrants enter at rate $M$, and have mean $z_{s}=-2.11$ replicate simulations were made, starting with a single migrant, and with a total of 55,000 generations. Over this time, 8 of the 11 replicates jumped to high density. Top left: The distribution of $N$, compared with the diffusion approximation (red), assuming $2 V^{*}=1$. Top middle: The trait mean, conditional on $N$. The black line shows the mean for each value of $N$, and is compared with the diffusion approximation (red curve). Top right: The variance of the mean, conditional on $N$, compared with the diffusion approximation (red curve). Dots show the variance, pooled into windows of width $\Delta N=10$. Bottom left: within-population variance, conditional on $N$. The black line shows the mean for each value of $N$, and is compared with the diffusion approximation (red curve). Bottom right: Mean identity, $\bar{F}$, conditional on $N$; this is used to calculate the predicted variance, $2 V^{*}$ (red line at bottom left). 
long-term genetic contribution, normalised so that the mean contribution of native individuals is one. When averaged over the trait values and degree of relatedness of migrants, this gives the gene flow factor; this is the ratio between the effective and the actual migration rates. This determines the strength of reproductive isolation, and hence, whether the new population should be considered a biological species.

This calculation is similar to Huisman and Tufto (2013), who make numerical calculations of the effective migration rate, under the infinitesimal model, assuming stabilising selection. They show that a simple approximation (Huisman and Tufto 2013, Eq. 16), based on the mean fitness of migrants versus natives, can be quite accurate, even though it does not account for changes in fitness over successive generations.

The new population will initially be highly inbred (Fig. 2), and gene flow may play an important role by introducing new genetic variation. Because individuals that migrate into a large population contribute additively to the decrease in the mean relatedness, $\bar{F}$, the rate of influx of trait variance due to migrants is proportional to their reproductive value, just as for allele frequencies or trait means. In the following, we describe how reproductive value depends on trait mean and on relatedness, assuming that the new population is at an equilibrium involving mutation and directional selection. We also outline the corresponding derivation for stabilising selection, but we are unable to provide an analytic expression for the reproductive value in this case. Derivations are in Appendix C.

\section{Directional selection}

Suppose that the newly established population is at equilibrium in a balance between directional selection and mutation. As before, we write $V$ for the variance in the trait among offspring of two unrelated individuals, the within-family variance is $V\left(1-F_{*}\right)$ where $F_{*}$ is the average identity of two individuals chosen from the population, and under directional selection with fitness $e^{\beta z}$ the equilibrium genetic variance is $2 V^{*}$.

Pragmatically, mutation can be incorporated into the infinitesimal model by scaling the recursion for trait values across generations to account for alleles that are replaced by mutants and by adding constants to both the trait mean and variance to account for the mutant alleles. In Barton et al. (2017) it is shown that this is consistent with 'house of cards' mutation in a model of an additive trait determined by a large number of Mendelian factors. Here we shall not model mutation explicitly. In each generation, selection increases the mean by $2 \beta V^{*}$, and we assume that this is counterbalanced by mutation. We assume that mutation only slowly increases the genetic 
variance, and that this is offset by weak random drift; these effects are assumed to be negligible on the short timescale set by recombination and by selection on the trait mean.

We define $w\left[z, F_{w}, F_{b}\right]$ to be the reproductive value of an individual backcrossing into the population. Here $z$ is the value of the trait of the individual, $F_{w}$ is the probability of identity of two distinct copies of a gene sampled within the individual, and $F_{b}$ is the mean identity between the individual and a randomly chosen individual from the established population.

First we consider a single generation. The immediate fitness of an individual is the expected number of its offspring, normalised by the average growth rate across the native population (given by Eq. 3). For an individual with trait value $z$, the immediate fitness is

$$
\begin{aligned}
\exp \left(-\beta \bar{z}-\beta^{2} V^{*}\right) \mathbb{E}\left[\exp \left(\beta \frac{(z+Y)}{2}+\frac{\beta^{2} V_{b}}{2}\right)\right] \\
=\exp \left(\beta \frac{(z-\bar{z})}{2}-\frac{\beta^{2} V^{*}}{4}+\frac{\beta^{2}}{2}\left(V_{b}-V^{*}\right)\right),
\end{aligned}
$$

where the expectation is over the trait value $Y$ of a randomly chosen mate from the native population and $V_{b}$ is the within-family variance among offspring:

$$
V_{b}=V\left(1-\frac{F_{w}+F_{*}}{2}\right) .
$$

For a native individual, $V_{b}=V^{*}$. For a migrant, we see two conflicting forces; we expect that for a new migrant $z<\bar{z}$, but if it brings extra variance $\left(V_{b}>V^{*}\right)$, the offspring can nonetheless be successful.

After selection and mutation (which reduces the mean by $2 \beta V^{*}$ ), the trait distribution of surviving offspring is normal with mean

$$
\frac{z+\bar{z}}{2}+\beta V_{b}-\frac{3 \beta V^{*}}{2}
$$

and variance $V_{b}+V^{*} / 2$. Writing $\mathcal{N}\left[x \mid \mu, \sigma^{2}\right]$ for the density function at $x$ of a normally distributed random variable with mean $\mu$ and variance $\sigma^{2}$, we now have a recursion for the reproductive value:

$$
\begin{aligned}
& w\left[z, F_{w}, F_{b}\right]=\exp \left(\beta \frac{(z-\bar{z})}{2}-\frac{\beta^{2} V^{*}}{4}+\frac{\beta^{2}}{2}\left(V_{b}-V^{*}\right)\right) \\
& \times \int w\left[x, F_{b}, \frac{F_{b}+F_{*}}{2}\right] \mathcal{N}\left[x \mid \frac{z+\bar{z}}{2}+\beta V_{b}-\frac{3 \beta V^{*}}{2}, V_{b}+\frac{V^{*}}{2}\right] d x .
\end{aligned}
$$


We solve this recursion in Appendix C.

For a native individual, with trait value $z, F_{w}=F_{b}=F_{*}$ and the reproductive value is

$$
w_{*}[z]=e^{\beta(z-\bar{z})-\beta^{2} V^{*}} .
$$

(Notice that this averages to one across native individuals.) Equation (15) says that the reproductive value is proportional to the square of the immediate fitness. This can be understood using Robertson's (1960) argument, that with no linkage, an inherited increase in fitness halves across each generation, and so persists for, on average, two generations. The fixation probability of a favourable mutation has the same dependence on reproductive value (Barton, 2009).

The reproductive value of a migrant individual with trait $z$ is

$$
w\left[z, F_{w}, F_{b}\right]=\exp \left(\beta(z-\bar{z})+4 \beta^{2}\left(V_{b}-V^{*}\right)-\beta^{2} V^{*}\right) .
$$

This decreases with $F_{w}, F_{b}$, because an inbred migrant has less variable offspring $\left(V_{b}<V^{*}=V\left(1-F_{*}\right)\right)$. The factor $\exp (z-\bar{z})$, which reduces the contribution of a less well-adapted migrant, is multiplied by a factor which increases the contribution if the migrant produces more variable offspring than the natives. When the new population is relatively outbred (right of Fig. 8), the effect of the trait mean dominates, and introgression is reduced. However, when it is highly inbred $\left(F_{*} \sim 1\right.$; left of Fig. 8$)$, the increased variance of hybrids can give them a strong advantage if there is a high genetic variance in the source population.

\section{Stabilising selection}

We now turn to the case of stabilising selection. We assume that the population is at equilibrium with constant relatedness $F_{*}$ between all genomes; fitness is proportional to $\exp \left(-s\left(z-z_{\mathrm{opt}}\right)^{2} / 2\right)$, and, since migration is assumed weak, relative to the size of the native population, the mean is assumed to be at the optimum (c.f. Eq. 13). The trait $z$ is taken to be normally distributed with variance $2 \hat{V}$. As before, $V^{*}=V\left(1-F_{*}\right)$ denotes the within family variance before selection. Because stabilising selection causes negative linkage disequilibrium, we will have $\hat{V}<V^{*}$ at equilibrium. In Appendix C we find an expression for $\hat{V}$ and show that for small $s$ it is approximately equal to $V^{*}$.

Before the action of selection, the offspring of two individuals sampled at random from the population have mean $z_{\text {opt }}$ and variance $\hat{V}+V^{*}$ and so an easy calculation (Appendix $\mathrm{C}$ ) shows that the mean growth rate across 


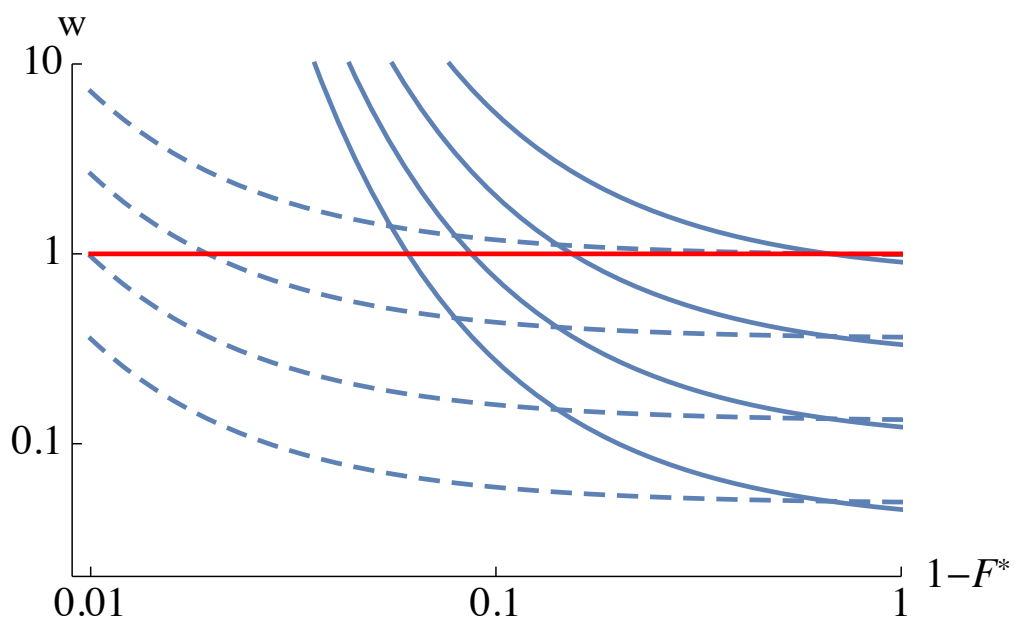

Figure 8: Reproductive value of an unrelated and outbred individual $\left(F_{w}=F_{b}=0\right)$ that backcrosses into a population with inbreeding coefficient $F_{*}$ under directional selection (Eq. 16). This population has genetic variance $2 V^{*}=2 V\left(1-F_{*}\right)$. The variance in $\log$ fitness is $2 \beta^{2} V_{*}=0.02$ (dashed curves), 0.2 (solid curves); relative fitness of the migrant is $e^{\beta(z-\bar{z})}=1, e^{-1}, e^{-2}, e^{-3}(=1,0.37,0.135,0.050)$ (top to bottom).

the population is $1 / \sqrt{1+s\left(\hat{V}+V^{*}\right)}$. Now focus on an individual with trait value $z$. The variance across its offspring (before selection) when it reproduces with a randomly chosen member of the native population is $\hat{V} / 2+V_{b}$, with $V_{b}$ given by (14). Normalising by the mean growth rate, an analogous calculation shows that the immediate fitness of the individual is

$$
\frac{\sqrt{1+s\left(\hat{V}+V^{*}\right)}}{\sqrt{1+s\left(\hat{V} / 2+V_{b}\right)}} \exp \left(-\frac{\left(z-z_{\text {opt }}\right)^{2}}{4} \frac{s}{2\left(1+s\left(\hat{V} / 2+V_{b}\right)\right)}\right) \text {. }
$$

The distribution of surviving offspring of this individual is normal with mean

$$
z_{\mathrm{opt}}+\frac{z-z_{\mathrm{opt}}}{2\left(1+s\left(\hat{V} / 2+V_{b}\right)\right)}
$$

and variance

$$
\frac{\hat{V} / 2+V_{b}}{1+s\left(\hat{V} / 2+V_{b}\right)},
$$

from which we deduce the recursion for the reproductive value. Using the 
same notation as for directional selection:

$$
\begin{gathered}
w\left[z, F_{w}, F_{b}\right]=\frac{\sqrt{1+s\left(\hat{V}+V^{*}\right)}}{\sqrt{1+s\left(\hat{V} / 2+V_{b}\right)}} \exp \left(-\frac{\left(z-z_{\text {opt }}\right)^{2}}{4} \frac{s}{2\left(1+s\left(\hat{V} / 2+V_{b}\right)\right)}\right) \\
\times \int \mathcal{N}\left[x \mid z_{\text {opt }}+\frac{\left(z-z_{\text {opt }}\right)}{2\left(1+s\left(\hat{V} / 2+V_{b}\right)\right)}, \frac{\left(\hat{V} / 2+V_{b}\right)}{\left(1+s\left(\hat{V} / 2+V_{b}\right)\right)}\right] w\left[x, F_{b}, \frac{F_{b}+F_{*}}{2}\right] d x .
\end{gathered}
$$

In general, even for native individuals we have not been able to find a closed form solution for this recursion. However, for small $s$, for a native individual, an approximate solution to (17) is provided by

$$
w(z) \propto \exp \left(-\theta \frac{s}{2}\left(z-z_{o p t}\right)^{2}\right),
$$

where

$$
\theta=\frac{-(2 \alpha-1)+\sqrt{4 \alpha^{2}+4 \alpha-15}}{4\left(\hat{V}+2 V^{*}\right)},
$$

and $\alpha=2+s\left(\hat{V}+2 V^{*}\right)$. Since for small $s$, at equilibrium, $\hat{V} \sim V^{*}$, substituting we find

$$
\theta \sim \frac{s}{3} \quad \text { as } s \downarrow 0,
$$

so that for small $s$ the reproductive value of a native individual is approximately proportional to the immediate fitness to the power $4 / 3$.

More generally, the reproductive value of migrants, and hence their contribution to gene flow, depends on their degree of inbreeding $\left(F_{w}\right)$, their relatedness to the new population $\left(F_{b}\right)$, and on the product of the strength of stabilising selection and the genetic variance, $2 s \mathrm{~V}$, which is a measure of the genetic load due to stabilising selection. The reproductive value, of an individual with the optimal trait value, $z=z_{\text {opt }}$, is greater than 1 if it comes from the native population, so that $F_{w}=F_{b}=F_{*}$, and increases with $2 s V$. Unrelated individuals that move into a highly inbred population have substantially lower reproductive value, because their offspring are highly variable, and hence tend to be further from the optimum.

\section{Discussion}

We analyse a simple, yet quite general, model for establishment in a new habitat, following the joint evolution of the numbers and fitness distribution 
of individuals in the new environment. Although most of our results come from simulations, we obtain approximations for the dynamics of establishment assuming moderately high migration rates, and for the reproductive isolation of an established population provided that the number of migrants per generation is much less than the equilibrium population size. More important, a thorough description is possible because there are just three parameters: the number of migrants per generation, $M$, and the mean and variance of log fitness of individuals from the source population, measured in the new environment. These quantities are, in principle, directly observable - though it has been very difficult to measure the genetic variance in fitness in any organism (Kruuk et al., 2014).

In our simulations, we see that the probability that a single migrant will establish a new population depends primarily on how many genetic standard deviations the source lies below the threshold fitness needed for positive growth $\left(\bar{z}_{s} / \sqrt{2 V}\right)$. Roughly, establishment becomes feasible if the mean of the source population is less than $\sim 4$ standard deviations below the threshold growth rate; even though individuals that would have positive growth may be extremely rare, their offspring will be selected for faster growth, allowing establishment. A positive growth rate is achieved in roughly equal measure by the success of migrants that by chance are better adapted to the new environment, and by the subsequent adaptation of their offspring. Following intense inbreeding during establishment, some genetic variance remains, but the extent of inbreeding - and hence, of future adaptation - is highly variable (Fig. 2).

Steady immigration gives more opportunity for establishment, and maintains higher genetic variance in the new population. However, it also pulls the diverging population back towards the maladaptation of the source; if the divergence of the source is greater than some threshold number of standard deviations, then the expected time to establishment increases indefinitely with migration rate, $M$ (Fig. 4, upper right). This threshold can be predicted by a simple deterministic approximation that ignores random fluctuations, and assumes a constant genetic variance (Fig. 5). Above this threshold level of divergence, establishment is still possible, provided that $M$ is not too high, but relies on random fluctuations that allow escape from a metastable 'sink' state.

We introduce a simple diffusion approximation, which captures the qualitative behaviour of the model. The key assumptions are that the genetic variance is constant - reasonable for moderate to large numbers of migrants - and that changes are slow. This continuous time approximation is the main source of error, since the small initial population size and strong fit- 
ness differences necessarily set a short timescale. Nevertheless, the diffusion approximation is a powerful aid to intuition, and readily extends to give the joint distribution of population size and trait means or allele frequencies in a population that experiences density regulation and stabilising selection (Eq. 12; see Banglawala, 2010).

We have used a very simple model of trait evolution, which depends primarily on a single parameter - the additive genetic variance. There is obvious scope for including greater realism - in particular, investigating the effects of the number of loci, and including dominance and inbreeding depression. The diffusion approach may be fruitful here, since it readily extends to discrete biallelic loci, with dominance (see Banglawala, 2010). Gomulkiewicz et al. (2010) argue that establishment is harder when more loci are involved. However, as they point out, this argument depends on which quantities are held fixed. If the additive variance is fixed, then there is a well-defined infinitesimal limit, which is insensitive to the number of loci and their distribution of effects.

Gomulkiewicz and Houle (2009) investigate the effects of genetic constraints on evolutionary rescue, using a model of stabilising selection on multiple traits. A large population will always reach the optimum, provided there is at least some genetic variance in every direction. However, when adaptation must occur rapidly if a small population is to survive, genetic constraints (represented by directions in trait space with low variance) may lead to extinction. Here, we only consider a single trait, which can be seen as the vector that connects the source population with the new optimum. Genetic constraints are represented by the variance along this direction. This will be a good approximation if the new optimum is far from the threshold for positive growth - a considerable simplification.

The rapid adaptation that is needed to establish a growing population in a new habitat is unlikely to depend on mutation: a heterozygote for a single mutation would need to increase growth rate to a positive value by itself. This may be plausible for specific environmental challenges - pesticides or plant toxins for example - but seems unlikely where multiple adaptations are needed for growth. However, a mutation of large effect could act together with polygenic adaptation. For a single migrant that carries such an allele in heterozygous form, it is trivial to extend our analysis. However, continued migration would tend to swamp the new allele. Indeed, the facilitating mutation might eventually be lost, even if its initial presence was crucial for establishment (c.f. Gomulkiewicz et al., 2010).

The strong dependence of establishment probability on the number of standard deviations that separate the source from the threshold for positive 
growth simply reflects the rapid decline of probability in the tails of a normal distribution. It implies that if the size of the gap, measured in standard deviations, fluctuates in space or time, then rare instances when the gap is small will be most likely to lead to establishment. Once a large population is established, it can increase and adapt further, until numbers are limited by competition, and the trait is limited by either stabilising selection, mutation pressure, or lack of variation that can further increase fitness.

Once a new population is established in large numbers, $N$, the effects of immigration on the overall trait distribution in the native population will be low if $M \ll N$. Moreover, immigrants will have low fitness in the new habitat; under the infinitesimal model with initial fitness $e^{\beta z}<1$, the rate of gene flow into the new population is given by Eq. (10). If gene flow is sufficiently reduced, then we have a model of speciation. Recalling the scenario in which demes in a metapopulation experience a fluctuating environment, whether the new population survives and remains distinct depends more on the distribution of suitable habitat than on genetic details.

Note that this mechanism is distinct from classical 'founder-effect' speciation, because it is impeded rather than assisted by inbreeding and the consequent loss of genetic variance during establishment (Barton and Charlesworth, 1984). Directional selection acts throughout establishment in the new environment, so that no fitness valley needs to be crossed. Nevertheless, a combination of genetic and demographic fluctuations are needed to establish large numbers of well-adapted individuals despite gene flow from populations that are already abundant in a different environment (or environments).

It is not immediately obvious whether the model analysed here should be classed as sympatric or parapatric. Microhabitats might be intimately mingled, and individuals from the source population might land randomly across habitats. However, individuals that do manage to survive in the new habitat are assumed to leave offspring there, and they must mate primarily with others born there. Provided that the original population is sufficiently many standard deviations from the threshold for growth in the new habitat, only a few individuals can immigrate for establishment to be feasible. So, this is a model of parapatric speciation. However, if the evolving trait itself induced habitat or mate preference, or a trait that determined premating isolation were added, we would have a model for sympatric speciation (c.f. Polechová and Barton, 2005; Barton, 2010). This could be seen as an extension of the infinitesimal models of Polechová and Barton (2005) and Barton (2010) to include local adaptation and random drift. 


\section{References}

Banglawala, N., 2010. Local adaptation under demographic and genetic fluctuations. $\mathrm{Ph} \mathrm{D}$ thesis, University of Edinburgh; http://hdl.handle.net/1842/4896.

Barton, N. H., 2009. Why sex and recombination? Cold Spring Harbor Symp. Quant. Biol 74, 187-195.

Barton, N. H., 2010. What role does natural selection play in speciation? Phil. Trans. Roy. Soc. London B 365, 1825-1840.

Barton, N. H., Charlesworth, B., 1984. Genetic revolutions, founder effects and speciation. Annu. Rev. Ecol. Evol. Syst. 15, 133-164.

Barton, N. H., Etheridge, A. M., Véber, A., 2017. The infinitesimal model: definition, derivation, and implications. Theor. Pop. Biol., http://dx.doi.org/10.10.16/j.tpb.2017.06.001.

Charlesworth, B., 2015. Causes of natural variation in fitness: Evidence from studies of Drosophila populations. Proc. Nat. Acad. Sci. U.S.A. 112 (6), 1662-1669.

Feller, W., 1951. Diffusion processes in genetics. Proc. Second Berkeley Symp., 227-246.

Gardiner, C., 2004. Stochastic Methods: A handbook for the natural and social sciences. Fourth Edition. Springer series in synergetics.

Gomulkiewicz, R., ad M Barfield, R. D. H., Nuismer, A. L., 2010. Genetics, adaptation, and invasion in harsh environments. Evolutionary applications $3,97-108$.

Gomulkiewicz, R., Holt, R. D., 1995. When does evolution by natural selection prevent extinction? Evolution 49, 201-207.

Gomulkiewicz, R., Houle, D., 2009. Demographic and genetic constraints on evolution. American Naturalist 174, E218-E229.

Gomulkiewicz, R., Shaw, R. G., 2013. Evolutionary rescue beyond the models. Phil. Trans. Roy. Soc. London B 368:20120093.

Haldane, J. B. S., 1956. The relation between density regulation and natural selection. Proc. Roy. Soc. London B 145, 306-308. 
Hill, W. G., Goddard, M. E., Visscher, P. M., 2008. Data and theory point to mainly additive genetic variance for complex traits. PloS Genetics 4 (2), e1000008. doi:10.1371/journal.pgen.1000008.

Holt, R. D., Barfield, M., 2011. Theoretical perspectives on the statics and dynamics of species' borders in patchy environments. American Naturalist 178, S6-S25.

Holt, R. D., Gomulkiewicz, R., 1997. How does immigration influence local adaptation? a reexamination of a familiar paradigm. Am. Nat. 149, 563572 .

Holt, R. D., Gomulkiewicz, R., Barfield, M., 2003. The phenomenology of niche evolution via quantitative traits in a 'black hole' sink. Proc. Roy. Soc. London B 270, 215-224.

Huisman, J., Tufto, J., 2001. When sources become sinks: migrational meltdown in heterogeneous habitats. Evolution 55, 1520-1531.

Huisman, J., Tufto, J., 2013. Modelling wild-domestic interbreeding: how selection on a quantitative trait affects gene flow at a neutral locus. J. Theor. Biol. 332, 42-51.

Kaweckki, T. J., 2008. Adaptation to marginal habitats. Annu. Rev. Ecol. Syst. 39, 321-342.

Kesten, H., 1970. Quadratic transformations: A model for population growth. Adv. Appl. Probab. 2, 1-82, 179-228.

Kesten, H., 1971. Some nonlinear stochastic growth models. Bull. Amer. Math. Soc. 77 (4), 492-511.

Kimura, M., 1981. Possibility of extensive neutral evolution under stabilizing selection with special reference to nonrandom usage of synonymous codons. Proc. Nat. Acad. Sci. U.S.A. 78, 5773-5777.

Kirkpatrick, M., Barton, N. H., 1997. Evolution of a species' range. American Naturalist 150, 1-23.

Kirkpatrick, M., Barton, N. H., 2006. Chromosome inversions, local adaptation and speciation. Genetics 173, 419-434.

Kruuk, L. E. B., Garant, D., Charmantier, A., 2014. Quantitative Genetics in the Wild. Oxford University Press. 
Le Corre, V., Kremer, A., 2012. The genetic differentiation at quantitative trait loci under local adaptation. Mol. Ecol. 21, 1548-1566.

Lynch, M., Walsh, B., 1998. Genetics and analysis of quantitative traits. Vol.1. Sinauer Press.

Polechova, J., Barton, N. H., 2005. Speciation through competition: a critical review. Evolution 59, 1194-1210.

Polechova, J., Barton, N. H., 2015. Limits to adaptation along environmental gradients. Proc. Nat. Acad. Sci. USA 112, 6401-6406.

Robertson, A., 1960. A theory of limits in artificial selection. Proc. Roy. Soc. London B 153, 234-249.

Tufto, J., 2000. Quantitative genetic models for the balance between migration and stabilising selection. Genet. Res. 76, 285-293.

Tufto, J., 2001. Effects of releasing maladapted individuals: a demographicevolutionary model. Am. Nat., 1-10.

Weber, K. E., Diggins, L. T., 1990. Increased selection response in larger populations. II. Selection for ethanol vapour resistance in Drosophila melanogaster at two population sizes. Genetics 125, 585-597.

Yeaman, S., 2015. Local adaptation by alleles of small effect. The American Naturalist 186, S74-S89.

\section{Appendix A. Gaussian approximations}

We develop a deterministic approximation which assumes that after migration, random mating, and selection, the population follows a Gaussian distribution. Otherwise, the derivation allows for strong selection, and for the excess variance generated by mixing populations. Our aim is to write down a recursion for the joint evolution of the population size, the mean trait value, and the variance within and between families. The distinction from the model developed in the main text is that here we also keep track of the evolution of the average identity in the new population. Figure A.9 compares the dynamics of the two approximations to simulations of the full infinitesimal model.

Recall the order of events over a generation in our infinitesimal model. At the start of the generation, $M$ migrants enter the population. Every pair 
Table A.2: Contributions from the three classes of mated pair.

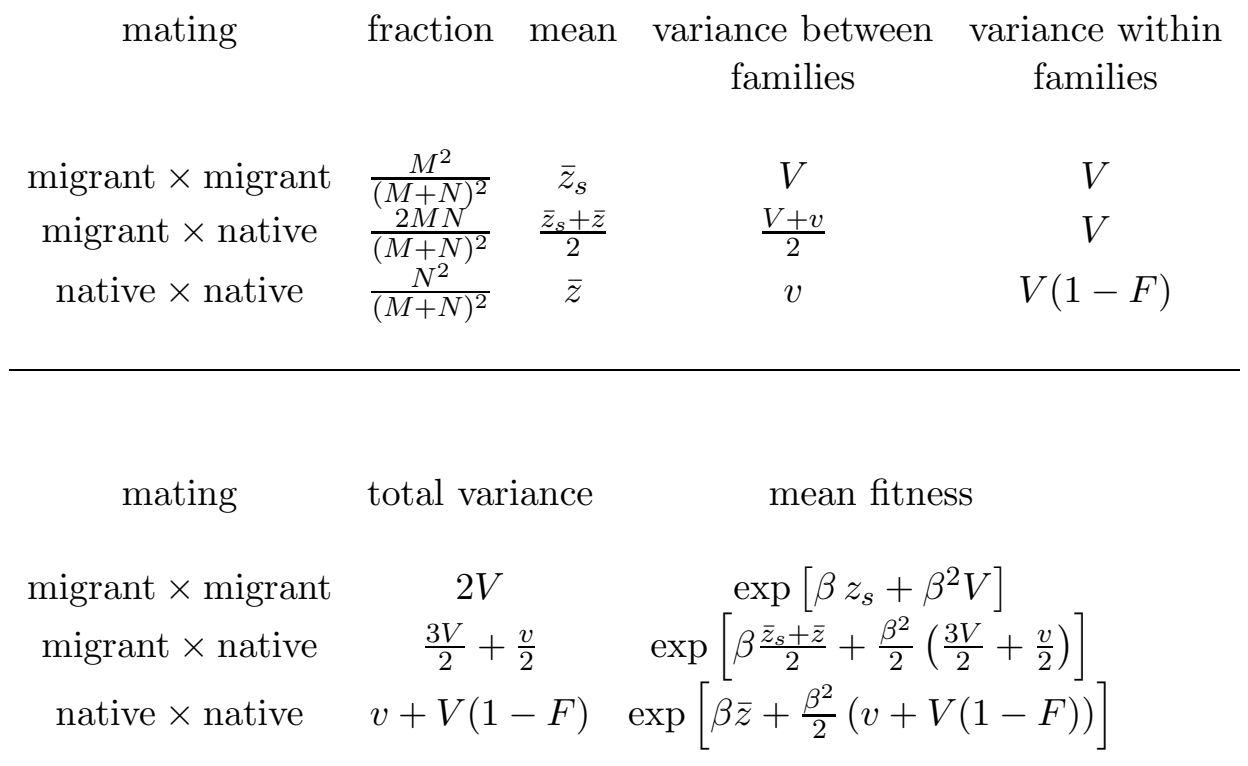

of individuals (both native and migrant) produces a random number of offspring with mean trait value the average of the two parents and (within family) variance determined by the relatedness between the parents. Offspring survive with a probability determined by their fitness. For our derivation it is convenient to follow the (equivalent) strategy of our simulations. Each pair of individuals in the population produces a Poisson number of offspring with mean $w_{i j}$ given by (2). The traits of offspring are independent samples from a normal distribution with mean $\beta v_{i j}+\left(z_{i}+z_{j}\right) / 2$, where $z_{i}$ and $z_{j}$ are the trait values of the parents and $v_{i j}$ is the within family variance.

Assume that the distribution of individuals in the population, size $N$, is Gaussian, with mean $\bar{z}$ and variance $2 v$. The average identity is $F$. At the start of the generation, $M$ migrants enter, with mean $\bar{z}_{s}$ and variance $2 V$; these make a fraction $m=\frac{M}{M+N}$ of the population. We now have three classes of mated pair (Table A.2).

The new population size, mean trait value among offspring before selec- 
tion, and the average within family variance are:

$$
\begin{aligned}
& N^{*}=\frac{M^{2}}{(M+N)} \exp \left(\beta \bar{z}_{s}+\beta^{2} V\right) \\
&+\frac{2 M N}{(M+N)} \exp \left(\beta \frac{\bar{z}_{s}+\bar{z}}{2}+\frac{\beta^{2}}{2}\left(\frac{3 V}{2}+\frac{v}{2}\right)\right) \\
&+\frac{N^{2}}{(M+N)} \exp \left(\beta \bar{z}+\frac{\beta^{2}}{2}(v+V(1-F))\right) ; \\
& N^{*} \bar{z}^{*}=\frac{M^{2}}{(M+N)} \exp \left(\beta \bar{z}_{s}+\beta^{2} V\right) \bar{z}_{s} \\
&+\frac{2 M N}{(M+N)} \exp \left(\beta \frac{\bar{z}_{s}+\bar{z}}{2}+\frac{\beta^{2}}{2}\left(\frac{3 V}{2}+\frac{v}{2}\right)\right) \frac{\left(\bar{z}+\bar{z}_{s}\right)}{2} \\
& \quad+\frac{N^{2}}{(M+N)} \exp \left(\beta \bar{z}+\frac{\beta^{2}}{2}(v+V(1-F))\right) \bar{z} ; \\
& N^{*} V_{w}^{*}=\frac{M^{2}}{(M+N)} V \exp \left(\beta \bar{z}_{s}+\beta^{2} V\right) \\
&+\frac{2 M N}{(M+N)}\left(\frac{3 V}{2}+\frac{v}{2}\right) \exp \left(\beta \frac{\bar{z}_{s}+\bar{z}}{2}+\frac{\beta^{2}}{2}\left(\frac{3 V}{2}+\frac{v}{2}\right)\right) \\
&\quad+V(1-F)) \exp \left(\beta \bar{z}+\frac{\beta^{2}}{2}(v+V(1-F))\right) .
\end{aligned}
$$

We shall add the between-class variance, and the change in mean trait value due to selection, later. Let $v=V+\delta \mathrm{v}$, and $\tilde{M}=M \exp \left(-\beta\left(\bar{z}-\bar{z}_{s}\right) / 2\right)$, $\tilde{N}=N \exp \left(\beta^{2} \delta v / 8\right)$. The expressions above become

$$
\begin{gathered}
N^{*}=\frac{\exp \left(\beta \bar{z}+\beta^{2} V\right)}{(M+N)}\left((\tilde{M}+\tilde{N})^{2}-\tilde{N}^{2} \gamma\right) ; \\
\left(\bar{z}^{*}-\bar{z}_{s}\right)=\frac{((\tilde{M}+\tilde{N})-\tilde{N} \gamma) \tilde{N}}{\left((\tilde{M}+\tilde{N})^{2}-\tilde{N}^{2} \gamma\right)}\left(\bar{z}-\bar{z}_{s}\right) ;
\end{gathered}
$$


and

$$
2 V_{w}^{*}=2 V+\frac{\tilde{N}(\tilde{M} \delta v+\tilde{N}(1-\gamma)(\delta v-V F))}{\left((\tilde{M}+\tilde{N})^{2}-\tilde{N}^{2} \gamma\right)}
$$

where

$$
\gamma=\left(1-\exp \left(-\beta^{2} V F / 2\right)\right) .
$$

We also need to find the identity, $F^{*}$, within the offspring, after selection. More precisely, we require the identity between the two distinct genes in a diploid individual, since this determines the segregation variance released by meiosis. Individuals from crosses between migrants and natives cannot be inbred; those from crosses amongst the $M$ migrants have identity $\frac{1}{2 M}$; and those from crosses amongst the $N$ natives have identity $\frac{(1-F)}{2 N}+F$. Hence

$$
F^{*}=\frac{1}{2 M} \phi_{M, M}+\phi_{N, N}\left(\frac{1}{2 N}(1-F)+F\right),
$$

where

$$
\phi_{N, N}=\frac{\tilde{N}^{2}(1-\gamma)}{\left((\tilde{M}+\tilde{N})^{2}-\tilde{N}^{2} \gamma\right)}, \quad \phi_{M, M}=\frac{\tilde{M}^{2}}{\left((\tilde{M}+\tilde{N})^{2}-\tilde{N}^{2} \gamma\right)} .
$$

The mean trait value of offspring will shift by $\beta$ times the within-family variance due to selection and so the shift in the mean trait value across the whole population due to selection is given by $2 \beta V_{w}^{*}$, so that after selection we have

$$
\left(\bar{z}^{* *}-\bar{z}_{s}\right)=\frac{((\tilde{M}+\tilde{N})-\tilde{N} \gamma)}{\left((\tilde{M}+\tilde{N})^{2}-\tilde{N}^{2} \gamma\right)} \tilde{N}\left(\bar{z}-\bar{z}_{s}\right)+2 \beta V_{w}^{*}
$$

Finally, we must add the variance generated by mixing the three classes. The three classes of mating are in the ratio $\alpha=\left\{\tilde{M}^{2}, 2 \tilde{M} \tilde{N}, \tilde{N}^{2}(1-\gamma)\right\}$, and have means (after selection) of $\mathbf{m}=\left\{0, \frac{\Delta}{2}+\beta \frac{\delta v}{2}, \Delta+\beta \delta v-F \beta V\right\}$, plus a constant; $\Delta=\bar{z}-z_{s}$. The variance generated by mixing the three classes is $\frac{1}{\left(\sum \alpha\right)^{2}}\left(\alpha \cdot \mathbf{m}^{2}\left(\sum \alpha\right)-(\alpha \cdot \mathbf{m})^{2}\right)$, where we have abused notation and 
written $\mathbf{m}^{2}$ for the vector whose entries are the squares of those of $\mathbf{m}$, giving:

$$
\begin{aligned}
V_{b}= & \frac{\tilde{M} \tilde{N}}{4\left((\tilde{M}+\tilde{N})^{2}-\tilde{N}^{2} \gamma\right)^{2}} \times\left\{2 A^{2}\left((\tilde{M}+\tilde{N})^{2}(1-\gamma)+\gamma \tilde{M}^{2}\right)\right. \\
& \left.-4 A \beta V F \tilde{N}(1-\gamma)(\tilde{M}+\tilde{N})+2 \beta^{2} V^{2} F^{2}(1-\gamma) \tilde{N}(\tilde{M}+2 \tilde{N})\right\},
\end{aligned}
$$

where $A=\beta \delta v+\left(\bar{z}-\bar{z}_{s}\right)$.

We can rewrite these expressions in a simpler dimensionless form by letting $y=\left(\bar{z}-\bar{z}_{s}\right) \sqrt{2 V}, \alpha=\beta \sqrt{2 V}, \delta=\delta v / V, \delta_{b}=V_{b} / V, n=N / M$, $\gamma=\left(1-\exp \left(-\alpha^{2} F / 4\right)\right.$ :

$$
\begin{gathered}
n^{*}=\frac{\exp \left(\beta \bar{z}_{s}+\frac{\alpha^{2}}{2}\right)}{(1+n)}\left((1+n C)^{2}-n^{2} C^{2} \gamma\right), \\
\delta_{w}^{*}=\frac{n C\left(\frac{\delta}{2}+n C(1-\gamma)\left(\frac{\delta}{2}-\frac{F}{2}\right)\right)}{\left((1+n C)^{2}-n^{2} C^{2} \gamma\right)}, \\
y^{* *}=\frac{(1+n C(1-\gamma))}{\left((1+n C)^{2}-n^{2} C^{2} \gamma\right)} n C y+\alpha\left(1+\delta_{w}^{*}\right), \\
\delta_{b}=\frac{n C}{4\left((1+n C)^{2}-n^{2} C^{2} \gamma\right)^{2}}\left\{2 a^{2}\left((1+n C)^{2}(1-\gamma)+\gamma\right)\right. \\
\left.-4 a \alpha F(1-\gamma) n C(1+n C)+\alpha^{2} F^{2}(1-\gamma) n C(1+2 n C)\right\}, \\
F^{*}=\frac{1}{2 M} \phi_{M, M}+\phi_{N, N} F\left(1-\frac{1}{2 M n}\right) \\
=\frac{1}{\left((1+n C)^{2}-n^{2} C^{2} \gamma\right)}\left(\frac{1}{2 M}+n^{2} C^{2}(1-\gamma) F\left(1-\frac{1}{2 M n}\right)\right),
\end{gathered}
$$

where

$$
\phi_{M, M}=\frac{1}{\left((1+n C)^{2}-n^{2} C^{2} \gamma\right)}, \quad \phi_{N, N}=\frac{n^{2} C^{2}(1-\gamma)}{\left((1+n C)^{2}-n^{2} C^{2} \gamma\right)},
$$

$\tilde{N} / \tilde{M}=n C$ and $C=\exp \left(\alpha y / 2+\alpha^{2} \delta / 8\right), a=y+\alpha \delta / 2$. If F reaches a 
quasi-equilibrium, $n^{*}=n$, and

$$
F \sim \frac{1}{\left(2 M(1+2 n C)+n C^{2}(1-\gamma)\right)} .
$$

If $F$ is negligible, as it will be for large $M$, we can set $F, \gamma=0$, to obtain

$$
\begin{gathered}
n^{*}=\frac{(1+n C)^{2}}{(1+n)} \exp \left(\beta \bar{z}_{s}+\frac{\alpha^{2}}{2}\right), \quad y^{* *}=\alpha+\frac{n C}{(1+n C)}\left(y+\alpha \frac{\delta}{2}\right), \\
\delta^{*}=\delta_{w}^{*}+\delta_{b}^{*}=\frac{n C}{(1+n C)} \frac{\delta}{2}+\frac{n C}{2(1+n C)^{2}}\left(y+\alpha \frac{\delta}{2}\right)^{2} .
\end{gathered}
$$

Figure A.9 compares this deterministic recursion with stochastic simulations of the full model. The mean of the source population is sufficiently negative that there is a 'sink' state that can trap the population. The panels show the outcome for different starting points. If the mean, $\bar{z}_{s}$, is initially low, the population remains small (top row, $\bar{z}_{s}=-2.5,-2,-1.5$ ), whilst if it is sufficiently high, it quickly escapes (bottom row, $\bar{z}_{s}=-1.0,-0.5,0$ ). The full Gaussian approximation developed in this section (orange dots) fits closely to the mean of those populations that remain trapped (red line), whilst the cruder approximation outlined in the main text which assumes a constant variance fits less well (brown dots).

Next, we find the critical point above which escape is inevitable. Assuming that $y \gg \alpha \delta$, and that $\delta$ is rapidly pulled towards a quasi-equilibrium, we have for $\alpha y$ small

$$
\delta \sim \frac{n C y^{2}}{((2+n C)(1+n C)-\alpha y n C)} \sim \frac{n y^{2}}{(2+n)(1+n)} .
$$

If $y^{2} \ll n$, then $\delta$ is small, and

$$
n^{*} \approx \frac{(1+n C)^{2}}{(1+n)} \exp \left(\beta \bar{z}_{s}+\frac{\alpha^{2}}{2}\right), \quad y^{* *} \approx \alpha+\frac{n C}{(1+n C)} y .
$$

Setting $y^{* *}=y$ in (A.3), we see that at equilibrium $(1+n C)=y / \alpha$ and since, by definition, $C=\exp (\alpha y / 2)$, we can write this as $n=(y-$ $\alpha) \exp (-\alpha y / 2) / \alpha$. Setting $n^{*}=n$ in (A.3) and rearranging gives

$$
\beta \bar{z}_{s}=\log \left(\frac{(y-\alpha)}{y^{2}} e^{-\alpha y / 2}\left(\alpha+(y-\alpha) e^{-\alpha y / 2}\right)\right)-\frac{\alpha^{2}}{2} .
$$

This has a peak at some critical value of $\beta \bar{z}_{s}$, which determines the critical 

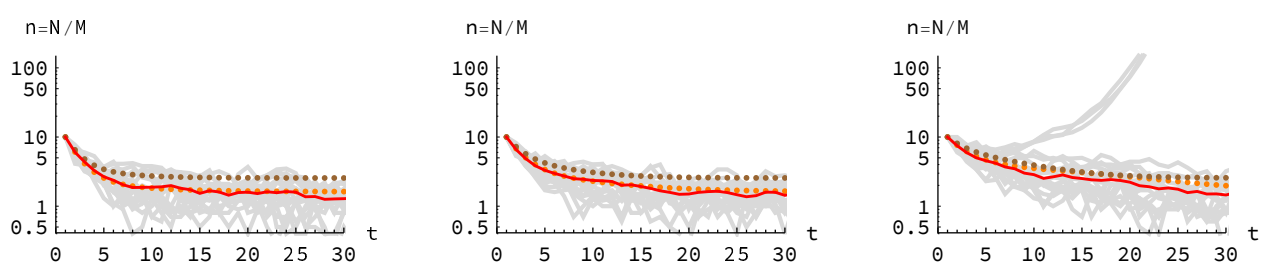

$\mathrm{n}=\mathrm{N} / \mathrm{M}$
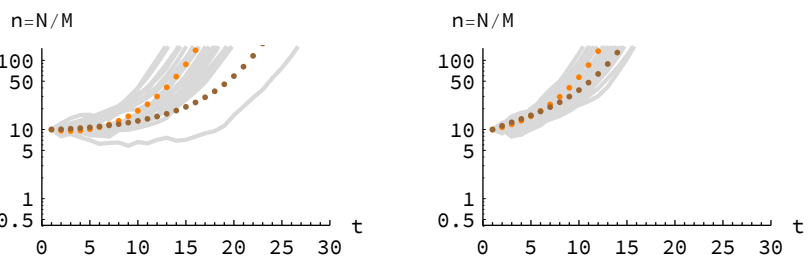

Figure A.9: We compare the recursions given by the Gaussian approximation of Appendix A with simulations of the full model; $\beta=0.25, M=10$. Grey lines show 20 replicates, starting from $N=100$, with the mean of the source population $\bar{z}_{s}=-2.5$. This is below the critical value of $\bar{z}_{s}=-2.0$ (from either Eq. 9 or Eq. A.4), and so there is an unstable point below which the population is trapped in a 'sink'. Each panel shows a different starting condition, $\bar{z}_{s}=-2.5,-2.0, \ldots, 0$ (from top left). Orange dots are the Gaussian approximation of Appendix A, whilst brown dots are the simpler approximation of Eqs. (4), (5), which assumes constant variance. The red line is the mean of those runs which did not escape by the end.

point below which a stable 'sink' state is possible.

The still simpler approximation developed in the main text assumes $C \sim$ 1, and approximates changes due to selection to be small, leading to Eqs. (8), (9). Figure A.10 shows that this is close to the Gaussian approximation of Eq. (A.4).

\section{Appendix B. A note on diffusion approximations}

In Section 4.2, we wrote down a system of differential equations to approximate the evolution of the population size and mean trait value, under the assumption that the trait value across the population has a Gaussian distribution with constant variance. If we wish the differential equations to mimic the discrete system given by equations (4) and (5), some care is needed in choosing the coefficients. 

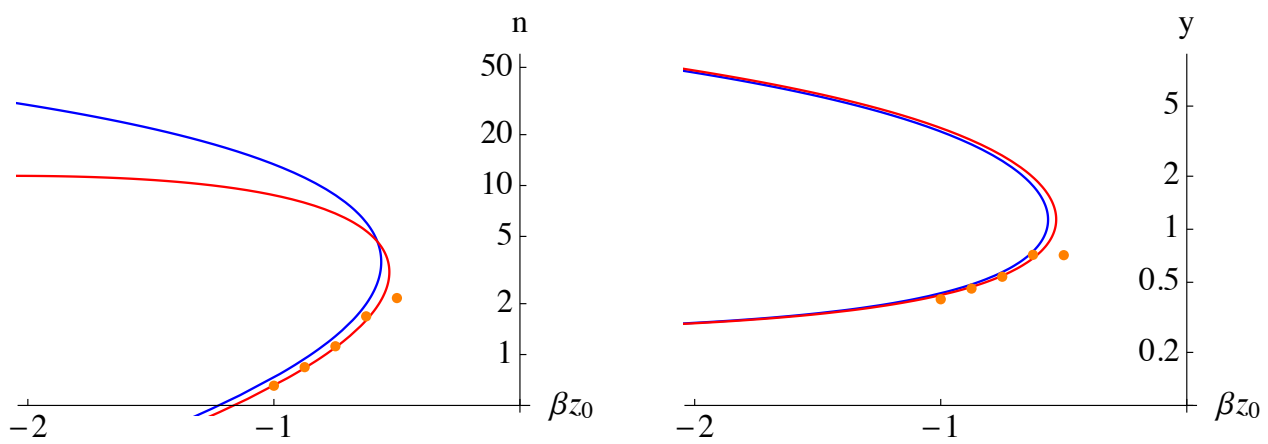

Figure A.10: This shows the equilibria for $n=\frac{N}{M}, y=\left(\bar{z}-\bar{z}_{s}\right) \sqrt{2 V^{*}}$ (left, right), against $\beta \bar{z}_{s}$, for $\beta=0.25$. The lower equilibrium is stable, the upper unstable; the critical value of $\beta z_{s}$ is where these equilibria merge. Blue: naive approximation (Eqs. 8,9); Red: Gaussian approximation (Eq. A.4). Orange points show means over simulations of the full model, run for $10^{4}$ generations, or until escape, and dropping the last 50 generations. This procedure was repeated until a total of at least 5000 generations were accumulated, summed over all runs.

It is convenient to consider the recursions for $N$ and $N \bar{z}$. These are:

$$
\begin{aligned}
N(t+1) & =M+N(t) \exp \left(\beta \bar{z}(t)+\beta^{2} V^{*}\right) \\
N(t+1) \bar{z}(t+1) & =\left(N(t) \bar{z}(t)+2 \beta V^{*} N(t)\right) \exp \left(\beta \bar{z}(t)+\beta^{2} V^{*}\right)+M \bar{z}_{s} .
\end{aligned}
$$

We note that the solution to

$$
\frac{d f(t)}{d t}=g(t)+a(t) f(t)
$$

satisfies

$$
f(t+T)=\int_{t}^{t+T} g(u) \exp \left(\int_{u}^{t+T} a(r) d r\right) d u+f(t) \exp \left(\int_{t}^{t+T} a(r) d r\right) .
$$

If we take the differential equations

$$
\begin{aligned}
& \frac{d N}{d t}=M+\beta \bar{z} N \\
& \frac{d \bar{z}}{d t}=2 \beta V^{*}\left(1-\frac{M}{2 N}\right)-\frac{M}{N}\left(\bar{z}-\bar{z}_{s}\right),
\end{aligned}
$$


then

$$
\frac{d(N \bar{z})}{d t}=M \bar{z}_{s}+2 \beta V^{*}\left(N-\frac{M}{2}\right)+\beta \bar{z}(N \bar{z}) .
$$

Solving for $N$ using (B.3) with $T=1$ yields:

$$
N(t+1)=\int_{t}^{t+1} M \exp \left(\int_{u}^{t+1} \beta \bar{z}(r) d r\right) d u+N(t) \exp \left(\int_{t}^{t+1} \beta \bar{z}(r) d r\right) .
$$

Now we expect $\bar{z}$ to change by order $\beta$ over a single generation (this will be the order of its shift due to selection) and so for small $\beta$, expanding the exponential, we see that

$$
N(t+1)=M+N(t)(1+\beta \bar{z}(t))+\frac{1}{2} \beta \bar{z}(t) M+\mathcal{O}\left(\beta^{2}(N(t)+M)\right) .
$$

Comparing with our discrete recursion (in which we also expand the exponential), the errors we are accumulating in using (B.4) in place of (B.1) are of order at most $\beta M+\beta^{2} N$ per generation. If we wanted to reduce the error to order $\beta^{2}(M+N)$, we would need to replace $M$ in (B.4) by $M(1-\beta \bar{z} / 2)$. The point is that in contrast to the discrete world, in which all migrants arrive after reproduction, here migrants arrive throughout the time interval and once arrived, they produce offspring, thereby increasing the overall effect of migrants on the population size at the end of the generation. We chose not to make this adjustment to $M$. We expect $\beta \bar{z} M$ to be small relative to $N$; once $\bar{z}$ is large, the population grows rapidly.

Now consider the equation for $N \bar{z}$. Again we use (B.3):

$$
\begin{aligned}
(N \bar{z})(t+1)=\int_{t}^{t+1}( & \left.M \bar{z}_{s}+2 \beta V^{*}\left(N(u)-\frac{M}{2}\right)\right) \\
\quad \times \exp \left(\int_{u}^{t+1} \beta \bar{z}(r) d r\right) d u & \quad+(N z)(t) \exp \left(\int_{t}^{t+1} \beta \bar{z}(r) d r\right) .
\end{aligned}
$$

Now again using (B.3), we see that for $u \in[t, t+1]$, up to an error of order 
$\beta, N(u)=N(t)+(u-t) M$. Substituting we find

$$
\begin{aligned}
(N \bar{z})(t+1)= & \int_{t}^{t+1}\left(M \bar{z}_{s}+2 \beta V^{*}\left(N(t)+M(u-t)-\frac{M}{2}\right)\right) \\
& \times \exp \left(\int_{u}^{t+1} \beta \bar{z}(r) d r\right) d u+(N z)(t) \exp \left(\int_{t}^{t+1} \beta \bar{z}(r) d r\right) \\
= & M \bar{z}_{s}\left(1+\frac{\beta \bar{z}}{2}\right)+2 \beta V^{*} N(t)+(N Z)(t)(1+\beta \bar{z}(t)) \\
& +\quad \mathcal{O}\left(\beta^{2}(M+N)\right) .
\end{aligned}
$$

Taking a factor $(1-M /(2 N))$ in (B.5) in place of the $(1-M / N)$ in the discrete equations, results in the cancellation of two terms $\beta V^{*} M$. Once again, we see that if we replace $M$ by $M(1-\beta \bar{z} / 2)$ in equation (B.4), our approximation is accurate up to order $\beta^{2}(M+N)$.

\section{Appendix C. Reproductive value of migrants}

\section{Directional selection}

Here we set up and solve a recursion for the reproductive value of an individual backcrossing into a population that has established in the new environment. We denote the reproductive value of an individual of trait $z$ by $w\left[z, F_{w}, F_{b}\right]$, where $F_{w}$ is the probability of identity of two distinct copies of a gene sampled within the individual and $F_{b}$ is the mean identity between the individual and a randomly chosen individual from the native population. In order to write down a recursion, we must keep track of not only the trait value, but also $F_{w}$ and $F_{b}$ for offspring of our chosen individual.

It is convenient to replicate the steps in our simulations. First we calculate the mean growth rate across the whole population. Then we calculate the contribution from our individual of trait $z$. Finally we write down the conditional distribution of the trait of a second individual sampled from the population, given that it produced an offspring with our chosen individual.

Since the population is assumed to be at equilibrium with mean $\bar{z}$ and variance $2 V^{*}$, exactly as before (Eq. 3), the mean growth rate of the population is $\exp \left(\beta \bar{z}+\beta^{2} V^{*}\right)$.

Suppose now that an individual has trait value $z$, and we calculate its expected number of offspring. Offspring could come from multiple partners in our model, but the mean number of offspring is

$$
w_{z}=\mathbb{E}\left[\exp \left(\beta \frac{(z+Y)}{2}+\frac{\beta^{2} V_{b}}{2}\right)\right]=\exp \left(\beta \frac{(z+\bar{z})}{2}+\frac{\beta^{2} V_{b}}{2}+\frac{\beta^{2} V^{*}}{4}\right),
$$


where the expectation on the left is with respect to the choice of the mate $Y$ from the population, and we have used $V_{b}$ to denote the within-family variance of the offspring:

$$
V_{b}=V\left(1-\frac{F_{w}+F_{*}}{2}\right) .
$$

The immediate fitness of the individual, that is its relative weight in the whole population, is

$$
\exp \left(\beta \frac{(z-\bar{z})}{2}-\frac{\beta^{2} V^{*}}{4}+\frac{\beta^{2}}{2}\left(V_{b}-V^{*}\right)\right) .
$$

To find the distribution of the trait $Y$ of the mate of our chosen individual, we must weight the random pick from the population by the relative fitness of offspring of parents $z$ and $y$ compared to the quantity $w_{z}$ that we just calculated. The resulting tilted distribution has density proportional to

$$
\exp \left(\beta \frac{(y-\bar{z})}{2}-\frac{(y-\bar{z})^{2}}{4 V^{*}}\right)
$$

and so is a normal distribution with mean $\bar{z}+\beta V^{*}$ and variance $2 V^{*}$. Given $Y=y$, the trait values among offspring will then be normal with mean $(z+y) / 2+\beta V_{b}-2 \beta V^{*}$, and variance $V_{b}$. The term $-2 \beta V^{*}$ in the mean reflects mutation (which restores the population to equilibrium).

Combining the above, we can now write down the recursion for the reproductive value. We write $\mathcal{N}\left[x \mid \mu, \sigma^{2}\right]$ for the density function at $x$ of a normally distributed random variable with mean $\mu$ and variance $\sigma^{2}$. Then

$$
\begin{aligned}
& w\left[z, F_{w}, F_{b}\right]=\exp \left(\beta \frac{(z-\bar{z})}{2}-\frac{\beta^{2} V^{*}}{4}+\frac{\beta^{2}}{2}\left(V_{b}-V^{*}\right)\right) \\
& \times \iint w\left[x, F_{b}, \frac{F_{b}+F_{*}}{2}\right] \mathcal{N}\left[\begin{array}{r}
x \\
\left.\mid \frac{y+z}{2}+\beta V_{b}-2 \beta V^{*}, V_{b}\right] \\
\times \mathcal{N}\left[y \mid \bar{z}+\beta V^{*}, 2 V^{*}\right] d y d x
\end{array}\right.
\end{aligned}
$$

We can find the reproductive value of a native individual as a special 
case. Writing $w_{*}[z]=w\left[z, F_{*}, F_{*}\right]$,

$$
\begin{aligned}
& w_{*}[z]=\exp \left(\beta \frac{(z-\bar{z})}{2}-\frac{\beta^{2} V^{*}}{4}\right) \\
& \times \iint w_{*}[x] \mathcal{N}\left[x \mid \frac{y+z}{2}-\beta V^{*}, V^{*}\right] \mathcal{N}\left[y \mid \bar{z}+\beta V^{*}, 2 V^{*}\right] d y d x
\end{aligned}
$$

To solve this, one can integrate out $y$ on the right hand side to see that $x$ has a normal distribution with mean $\left(z+\bar{z}-\beta V^{*}\right) / 2$ and variance $3 V^{*} / 2$ and then postulating a solution of the form $\exp \left(\theta \beta(z-\bar{z})-\theta^{2} \beta^{2} V^{*}\right)$ and matching coefficients yields

$$
w_{*}[z]=\exp \left(\beta(z-\bar{z})-\beta^{2} V^{*}\right) .
$$

(The second term in the exponent is needed in order for the mean reproductive value across the population to be one). That is the reproductive value is proportional to the square of the immediate fitness.

One can apply a similar technique to find the reproductive value of a migrant, but now we obtain a recursion. Again integrating out $y$ in equation (C.2), we find that $x$ is normally distributed with mean $(z+\bar{z}) / 2+$ $\beta V_{b}-3 \beta V^{*} / 2$, and variance $V_{b}+V^{*} / 2$. We make the ansatz that

$$
w\left[z, F_{w}, F_{b}\right]=\exp \left(\delta\left(F_{w}, F_{b}\right)(z-\bar{z})-\theta\left(F_{w}, F_{b}\right)\right) .
$$

Then equation (C.2) becomes

$$
\begin{aligned}
w\left[z, F_{w}, F_{b}\right] & =\exp \left(\beta \frac{(z-\bar{z})}{2}-\frac{\beta^{2} V^{*}}{4}+\frac{\beta^{2}}{2}\left(V_{b}-V^{*}\right)\right) \\
& \times \exp \left(\delta\left(F_{b}, \frac{F_{b}+F_{*}}{2}\right)\left(\frac{z-\bar{z}}{2}+\beta V_{b}-\frac{3 \beta V^{*}}{2}\right)\right. \\
& \left.+\delta\left(F_{b}, \frac{F_{b}+F_{*}}{2}\right)^{2} \frac{1}{2}\left(V_{b}+\frac{V^{*}}{2}\right)-\theta\left(F_{b}, \frac{F_{b}+F_{*}}{2}\right)\right) .
\end{aligned}
$$

Equating the exponents in (C.5) yields

$$
\delta\left(F_{w}, F_{b}\right)=\frac{\beta}{2}+\frac{1}{2} \delta\left(F_{b}, \frac{F_{b}+F_{*}}{2}\right),
$$

and iterating we see that

$$
\delta\left(F_{w}, F_{b}\right)=\beta
$$


Substituting $\delta\left(F_{w}, F_{b}\right)=\delta\left(F_{b},\left(F_{b}+F_{*}\right) / 2=\beta\right.$ into the recursion for $\theta$ obtained by equating coefficients in (C.5) we obtain

$$
-\left(\theta\left(F_{w}, F_{b}\right)-\theta\left(F_{b}, \frac{F_{b}+F_{*}}{2}\right)\right)=2 \beta^{2}\left(V_{b}-V_{*}\right)=\beta^{2} V\left(F_{*}-F_{w}\right) .
$$

Again iterating, and using the solution we obtained before for a native individual, we find that,

$$
\theta\left(F_{w}, F_{b}\right)=\beta^{2} V^{*}-4 \beta^{2}\left(V_{b}-V^{*}\right) .
$$

Thus the reproductive value of a migrant individual with trait $z$ is

$$
w\left[z, F_{w}, F_{b}\right]=\exp \left(\beta(z-\bar{z})+4 \beta^{2}\left(V_{b}-V^{*}\right)-\beta^{2} V^{*}\right) .
$$

\section{Stabilising selection}

We assume that the fitness is proportional to $\exp \left(-s\left(z-z_{\mathrm{opt}}\right)^{2} / 2\right)$ and the mean in the population is assumed to be at the optimum. Because stabilising selection causes negative linkage disequilibrium, the variance in the trait across the population will no longer be $2 V^{*}$ Let us write $2 \hat{V}$ for the variance across the whole population. We retain the notation $V^{*}=V\left(1-F_{*}\right)$ for within family variance (before selection), where $V$ is the variance (before selection) among offspring of two unrelated individuals.

Before the action of selection, the variance among offspring is $\widetilde{V}=\hat{V}+$ $V^{*}$. The action of selection reduces this to $\widetilde{V} /(1+s \widetilde{V})$ and so at equilibrium

$$
2 \hat{V}=\frac{\tilde{V}}{(1+s \widetilde{V})},
$$

leading to a quadratic in $\hat{V}$ whose positive solution is

$$
\hat{V}=\frac{1}{4 s}\left(\sqrt{1+12 s V\left(1-F_{*}\right)+\left(2 s V\left(1-F_{*}\right)\right)^{2}}-\left(1+2 s V\left(1-F_{*}\right)\right)\right)
$$

which tends to $V^{*}$ for small $s$. So for small $s$, the variance in trait values across the population is $\sim 2 V^{*}$, as for the classical infinitesimal model.

To set up a recursion for the reproductive value, it is convenient to note 
that if $Z$ is normally distributed with mean $\mu$ and variance $\sigma^{2}$, then

$$
\begin{aligned}
\mathbb{E}\left[\exp \left(-s \frac{Z^{2}}{2}\right)\right] & \\
=\int \frac{1}{\sqrt{2 \pi \sigma^{2}}} \exp \left(-\frac{s \sigma^{2}+1}{2 \sigma^{2}}\right. & \left.\left(z-\frac{\mu}{s \sigma^{2}+1}\right)^{2}-\frac{\mu^{2} s}{2\left(s \sigma^{2}+1\right)}\right) \\
& =\frac{1}{\sqrt{1+s \sigma^{2}}} \exp \left(-\frac{\mu^{2} s}{2\left(1+s \sigma^{2}\right)}\right) .
\end{aligned}
$$

First we calculate the mean growth rate in the population. Before selection, the distribution of the offspring of two individuals sampled at random from the population is Gaussian with mean $z_{\text {opt }}$ and variance $\hat{V}+V^{*}$ and so using (C.8), the mean growth rate is $1 / \sqrt{1+s\left(\hat{V}+V^{*}\right)}$.

Now, define $w\left[z, F_{w}, F_{b}\right]$ to be the reproductive value of an individual with trait value $z$ backcrossing into such a population. By analogy with our calculation for directional selection, our first task is to find its mean number of offspring. Before selection, the trait value of offspring is normal with mean $\left(z+z_{\text {opt }}\right) / 2$ and variance $\hat{V} / 2+V_{b}$ where, as before, $V_{b}$ is the within family variance of the offspring and is defined by (C.1). Using (C.8) applied to the offspring trait value minus $z_{\mathrm{opt}}$ which has mean $\left(z-z_{\mathrm{opt}}\right) / 2$ and variance $\hat{V} / 2+V^{*}$, and our expression for the growth rate across the population, the immediate fitness of the individual is

$$
\frac{\sqrt{1+s\left(\hat{V}+V^{*}\right)}}{\sqrt{1+s\left(\hat{V} / 2+V_{b}\right)}} \exp \left(-\frac{\left(z-z_{\mathrm{opt}}\right)^{2}}{4} \frac{s}{2\left(1+s\left(\hat{V} / 2+V_{b}\right)\right)}\right) \text {. }
$$

Now we can read off the distribution of the trait value of successful offspring from the calculation that led to (C.8). It is normal with mean

$$
z_{\mathrm{opt}}+\frac{\left(z-z_{\mathrm{opt}}\right)}{2\left(1+s\left(\hat{V} / 2+V_{b}\right)\right)}
$$

and variance $\left(\hat{V} / 2+V_{b}\right) /\left(1+s\left(\hat{V} / 2+V_{b}\right)\right)$. The recursion for the reproductive 
value is therefore

$$
\begin{gathered}
w\left[z, F_{w}, F_{b}\right]=\frac{\sqrt{1+s\left(\hat{V}+V^{*}\right)}}{\sqrt{1+s\left(\hat{V} / 2+V_{b}\right)}} \exp \left(-\frac{\left(z-z_{\mathrm{opt}}\right)^{2}}{4} \frac{s}{2\left(1+s\left(\hat{V} / 2+V_{b}\right)\right)}\right) \\
\times \int \mathcal{N}\left[x \mid z_{\mathrm{opt}}+\frac{\left(z-z_{\mathrm{opt}}\right)}{2\left(1+s\left(\hat{V} / 2+V_{b}\right)\right)}, \frac{\left(\hat{V} / 2+V_{b}\right)}{\left(1+s\left(\hat{V} / 2+V_{b}\right)\right)}\right] w\left[x, F_{b}, \frac{F_{b}+F_{*}}{2}\right] d x .
\end{gathered}
$$

We have been unable to find a closed form solution to this equation, even for native individuals. However, when $s$ is small, for a native individual it is close to Gaussian. To see why, try a solution of the form

$$
w_{*} \propto \exp \left(-\theta \frac{s}{2}\left(z-z_{\mathrm{opt}}\right)^{2}\right),
$$

then substituting in (C.9) with $F_{b}=F_{*}$ and equating exponents, we find a quadratic in $\theta$. Writing $\alpha=2+s\left(\hat{V}+2 V^{*}\right)$, the positive solution is

$$
\theta=\frac{-(2 \alpha-1)+\sqrt{4 \alpha^{2}+4 \alpha-15}}{4(\alpha-2)} .
$$

For small $s$, we saw above that at equilibrium $\hat{V} \sim V^{*}$ and substituting we find

$$
\theta \sim \frac{1}{3} \quad \text { as } s \downarrow 0 .
$$

Moreover, substituting back into the recursion, we see that with this value of $\theta$, as $s \downarrow 0$, we have an approximate solution. Thus, for small $s$, the reproductive value of a native individual is approximately proportional to the immediate fitness to the power $4 / 3$.

The reason that this approach does not work for larger values of $s$ is that although the same expression for $\theta$ in terms of $\alpha$ ensures that when we substitute $w_{*}$ of this form into (C.9), the exponents in the exponentials on both sides of the equations match, the constant terms multiplying those exponentials are not equal. 